\title{
Low-derivative operators of the Standard Model effective field theory via Hilbert series methods
}

\author{
Landon Lehman and Adam Martin \\ Department of Physics, University of Notre Dame, \\ Nieuwland Science Hall, Notre Dame, IN 46556, U.S.A. \\ E-mail: 1lehman@nd.edu, amarti41@nd.edu
}

ABSTRACT: In this work, we explore an extension of Hilbert series techniques to count operators that include derivatives. For sufficiently low-derivative operators, we conjecture an algorithm that gives the number of invariant operators, properly accounting for redundancies due to the equations of motion and integration by parts. Specifically, the conjectured technique can be applied whenever there is only one Lorentz invariant for a given partitioning of derivatives among the fields. At higher numbers of derivatives, equation of motion redundancies can be removed, but the increased number of Lorentz contractions spoils the subtraction of integration by parts redundancies. While restricted, this technique is sufficient to automatically recreate the complete set of invariant operators of the Standard Model effective field theory for dimensions 6 and 7 (for arbitrary numbers of flavors). At dimension 8, the algorithm does not automatically generate the complete operator set; however, it suffices for all but five classes of operators. For these remaining classes, there is a well defined procedure to manually determine the number of invariants. Assuming our method is correct, we derive a set of 535 dimension- $8 N_{f}=1$ operators.

Keywords: Gauge Symmetry, Effective field theories, Global Symmetries, Standard Model

ArXiv EPRINT: 1510.00372 


\section{Contents}

1 Introduction and Hilbert series review 2

2 Including derivatives in Hilbert series $\quad 6$

$\begin{array}{ll}2.1 \text { Example: complex scalar } & 10\end{array}$

$\begin{array}{ll}2.2 \text { Example: dimension-6 SMEFT } & 10\end{array}$

$\begin{array}{lll}2.3 & \text { Example: dimension-7 SMEFT } & 13\end{array}$

3 SMEFT: dimension-8 operators $\quad \mathbf{1 5}$

$\begin{array}{lll}3.1 & \text { Derivative-free operators } & 15\end{array}$

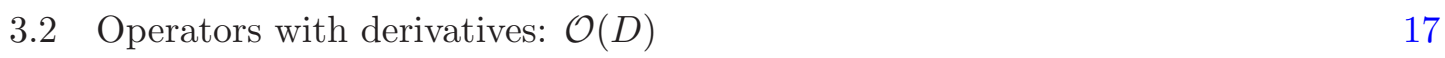

$\begin{array}{lll}3.3 & \text { Operators with derivatives: } \mathcal{O}\left(D^{2}\right) & 19\end{array}$

3.4 Operators with derivatives: $\mathcal{O}\left(D^{3}\right) \quad 22$

3.5 Operators with derivatives: $\mathcal{O}\left(D^{4}\right) \quad 22$

3.6 Total number of dimension-8 operators 23

4 Discussion $\quad 23$

$\begin{array}{ll}\text { A SMEFT fields and quantum numbers } & 25\end{array}$

B From Hilbert series to operators: adding indices $\quad 25$

C Hilbert series for a single scalar field $\quad 26$

$\begin{array}{ll}\text { D Dimension-7 operators for arbitrary } N_{f} & 27\end{array}$

In a previous paper [1], we reviewed the Hilbert series technique and highlighted its application for determining the complete set of operators in an effective field theory (EFT). We gave several examples, both using only Standard Model (SM) field content and also using beyond the Standard Model setups. All of the examples in [1], however, were restricted to non-derivative operators.

Operators with derivatives face two additional complications compared to operators without derivatives: redundancy due to the equations of motion (EOM), and redundancy due to integration by parts (IBP). Work on both of these issues has recently been presented in the context of scalars in $(0+1)$ dimensional spacetime [2]. Inspired by this success in lower dimensions, the goal of this present work is to extend the Hilbert series technique of invariant counting to operators with derivatives in four spacetime dimensions.

While a complete method for incorporating derivatives and their associated issues into Hilbert series for four-dimensional EFTs remains out of grasp, we have found an algorithm 
that seems to suffice for operators with low numbers of derivatives. Exactly what counts as a 'low-derivative' operator depends on the fields involved, but typically it means fewer than two derivatives. Before introducing our conjectured algorithm to incorporate derivatives into Hilbert series, we first briefly review the Hilbert series method and more clearly define the derivative issues in that context.

\section{Introduction and Hilbert series review}

The Hilbert series is defined as a power series

$$
\mathfrak{H}=\sum c_{n} t^{n}
$$

where $t^{n}$ indicates the invariants involving $n$ objects $t$, and $c_{n}$ is the number of independent invariants at that order. The Hilbert series technique has previously been used in wide-ranging theoretical contexts [3-18] and in more phenomenologically oriented applications [19-21], but the issues associated with derivatives have not yet been addressed. The phenomenological utility of this abstract mathematical object $\mathfrak{H}$ becomes clear once we identify $t$ with operators of some quantum field theory (QFT) and $n$ as the overall mass dimension of the operator; $\mathfrak{H}$ then gives the set of gauge, global, and Lorentz invariant operators of mass dimension $n$. Here $c_{n}$ tells us the number of independent invariants, but nothing about the values of the coefficients those invariants may have in the Lagrangian. These details depend on UV physics.

While Hilbert series techniques can be applied to any $\mathrm{QFT},{ }^{1}$ the application we are most interested in here is to count invariants of the Standard Model effective field theory (SMEFT). Given the absence of new light states in the first run of the LHC, the SMEFT is both a powerful and an agnostic way to bound new physics. Since the Higgs discovery in 2012 there has been intense work on the SMEFT on both the theoretical and experimental fronts [22-42]. This program will only increase in intensity as data from the second LHC run begins to accumulate, as higher statistics will allow more precise measurements and more (i.e. differential) observables. The invariant operators of the SMEFT are relatively straightforward to construct at low mass dimension, but the calculation becomes increasingly difficult at higher mass dimension. At mass dimension $d=5$ there is only 1 term [43], at $d=6$ there are $63[28,44-46]$, and at $d=7$ there are 20 [47]. No complete set of $d=8$ operators has been presented to our knowledge, although partial sets containing interactions of three neutral gauge bosons have been presented in [48, 49].

Once we recast the SMEFT (or any EFT) into the formalism of a Hilbert series, we can use powerful mathematical techniques to evaluate $\mathfrak{H}$. Specifically, $\mathfrak{H}$ can be written as the Haar integration over the relevant symmetry groups of a generating function known as the plethystic exponential (PE) $[4,6,7,10,18]$. The input to the $\mathrm{PE}$ is the field content of the theory, meaning the number of flavors and the quantum numbers of the fields in question.

\footnotetext{
${ }^{1}$ Provided the fields transform linearly under the symmetries of the theory, and the symmetry groups are compact Lie groups.
} 
The PE for a (bosonic) field $\phi$ in a representation $R$ of the symmetry group of the theory is formed by

$$
\mathrm{PE}\left[\phi_{R}\right]=\exp \left(\sum_{r=1}^{\infty} \frac{\phi^{r} \chi_{R}\left(z_{j}^{r}\right)}{r}\right),
$$

where $\chi_{R}$ is the character function of the representation $R$ expanded into monomials of $j$ complex variables, where $j$ is the rank of the group in question. As an example, for the SM Higgs field charged under $\mathrm{SU}(2)_{w} \otimes \mathrm{U}(1)_{Y}$, the entry in the above PE would be

$$
\phi \chi_{R}=H\left(z+\frac{1}{z}\right) u^{1 / 2}
$$

where $z$ is the complex variable parameterizing $\mathrm{SU}(2)_{w}$ and $u$ is the variable for $\mathrm{U}(1)_{Y}$. Here and throughout, $H$ is taken to be another complex variable of modulus $<1$ and not a full-fledged quantum field. We will refer to these complex numbers that represent fields as "spurions."

For anticommuting spurions $\psi$, which represent fermionic fields, the generating function is the fermionic plethystic exponential (PEF) [18], defined as

$$
\operatorname{PEF}\left[\psi_{R}\right]=\exp \left(\sum_{r=1}^{\infty} \frac{(-1)^{r+1} \psi^{r} \chi_{R}\left(z_{j}^{r}\right)}{r}\right) .
$$

The product of the PE for all bosonic spurions and the PEF for all fermionic spurions gives the complete generating function for the theory (which we call $\mathrm{PE}_{\text {tot }}$ ) at zeroth order in derivatives. Expanding, $\mathrm{PE}_{\text {tot }}$ generates all possible combinations of the input spurions. We can pick out the invariants of the symmetry group from the complete set of combinations by integrating over the Haar measure for each individual symmetry group included in $\mathrm{PE}_{\text {tot }}$. For the Higgs example above, the two individual groups are $\mathrm{SU}(2)_{w}$ and $\mathrm{U}(1)_{Y}$, but for the complete SMEFT this must be extended to include $\mathrm{SU}(3)_{c}$ and the Lorentz group as well. The wide variety of the SMEFT field content — fermions, scalars, gauge fields, chiral charges, vector charges, etc. is yet another reason to use it as a working example for illustrating Hilbert series methods.

Integrating over the Haar measure projects out the group invariants because the characters of a compact Lie group form an orthonormal basis for class functions, i.e. functions of the $j$ complex variables parameterizing the group: for group representations $R$ and $M$, $\int d \mu \chi_{R} \chi_{M}^{*}=\delta_{R M}$. As such, we can expand any function of the $j$ variables as a linear combination of characters:

$$
f(z)=\sum_{R} A_{R} \chi_{R}(z)
$$

where $A_{R}$ are coefficients that are independent of the complex number(s) used to parameterize the group ( $z$ in this case). Integrating $f(z)$ over the Haar measure, itself a function of $z$, projects out the part of $f$ in the trivial representation, $A_{0}$. In practice, each Haar integration can be expressed as a contour integral. (See appendix $\mathrm{C}$ for an example. Explicit expressions for the Haar measures of other simple Lie groups can be found in $[1,7]$.) Taking 
the residues of the poles in order to carry out this (potentially multiple) contour integral yields the number and form of the invariants of the theory. See ref. [1] for examples.

Our inclusion of Lorentz symmetry requires a more detailed explanation. Lorentz symmetry must be included as soon as we include particles with spin greater than zero, regardless of whether or not derivatives are added. The orthonormality of the characters just described only holds for compact Lie groups, and the Lorentz group is not compact. However, since we only care about operator counting and not about dynamics, we can work in Euclidean space where the Lorentz group is compact: $\mathrm{SO}(4) \cong \mathrm{SU}(2)_{R} \otimes \mathrm{SU}(2)_{L}$. For simplicity we work with fundamentally left-handed fields, defined to sit in the $(0,1 / 2)$ representation of $\mathrm{SU}(2)_{R} \otimes \mathrm{SU}(2)_{L}$; the hermitian conjugates are then in the $(1 / 2,0)$ representation. Gauge field strengths also transform under the Lorentz group, and we work with the combinations $X_{\mu \nu}^{L, R}=\frac{1}{2}\left(X_{\mu \nu} \pm i \tilde{X}_{\mu \nu}\right)$ (alternately referred to as $X^{-}$and $\left.X^{+}\right)$, which lie in the $(0,1)$ and $(1,0)$ representations respectively [29]. ${ }^{2}$

Applying the above discussion to the SMEFT PE, we can write $\mathrm{PE}_{\text {tot }}$ as a formal expansion in characters of all SM groups

$$
\mathrm{PE}_{\mathrm{tot}}\left[Q, u^{c}, d^{c}, \ldots, H, B^{L, R}, \ldots\right]=\sum_{G}\left(\sum_{R_{G}} A_{R_{G}} \chi_{R_{G}}\left(z_{G}\right)\right),
$$

where the index $G$ runs over the groups $\mathrm{SU}(2)_{L}, \mathrm{SU}(2)_{R}, \mathrm{SU}(3)_{c}, \mathrm{SU}(2)_{w}, \mathrm{U}(1)_{Y}$, and the index $R$ runs over the included representations within each group (singlet, doublet, triplet, etc.). The $A_{R_{G}}$ are functions of all SM fields (as spurions) alone, while the characters are functions of the complex numbers used to parameterize groups (the $z_{G}$ in eq. (1.6)). The full SMEFT Hilbert series at zeroth order in derivatives is the coefficient of the overall (gauge and Lorentz) singlet, which we extract by integrating the full plethystic exponential $\mathrm{PE}_{\text {tot }}$ over all of the group volumes:

$$
\mathfrak{H}_{\mathrm{SM}}=\int \prod_{G} d \mu_{G} \mathrm{PE}_{\mathrm{tot}}\left[Q, u^{c}, d^{c}, \ldots, H, B^{L, R}, \ldots\right]
$$

Finding all of the poles and associated residues of the Hilbert series - and therefore implicitly finding the number of invariants at all orders of mass dimension - is a daunting computational task, especially for an EFT with as many fields as the SMEFT. Instead of attacking the all-orders expression, we will expand $\mathfrak{H}$. Specifically, each field (spurion) is weighted by its mass dimension, i.e. $Q \rightarrow \epsilon^{3 / 2} Q, H \rightarrow \epsilon H$, etc., and we expand to the desired order in $\epsilon$ (for example, take the coefficient of $\epsilon^{6}$ for the calculation of the dimension- 6 operators). After expanding and picking out the desired coefficient, all of the poles of the integrand are at the origin of the complex plane, making the residues much easier to calculate. ${ }^{3}$

At a given order in mass dimension, the output of the Hilbert series is a sequence of spurions representing an invariant combination of fields. No information on how to actually

\footnotetext{
${ }^{2}$ Here $\tilde{X}_{\mu \nu}=\epsilon_{\mu \nu \rho \sigma} X^{\rho \sigma} / 2$.

${ }^{3}$ To apply these techniques in other spacetime dimensions, this mass counting would have to be altered along with the size of the Lorentz group.
} 
form the invariant (i.e. contract the group indices) is provided, so this must be inferred from context. For concreteness, we can carry out this reverse engineering for an example operator in the SMEFT in appendix B.

To extend this technique to include all physical operators in an EFT, we need to include derivatives. As mentioned earlier, derivatives bring two problems. First, if two operators differ only by terms that reduce upon using the equations of motion, they are redundant and only one should be included when counting invariants. ${ }^{4}$ The second problem occurs when two operators differ by a total derivative and can therefore be related using integration by parts. This is also a redundancy and only one operator should be kept in the physical basis. Often, operators are related by the combined action of both redundancies, i.e. IBP on one operator creates a second operator plus pieces that are proportional to EOM.

As an example, if we asked for all possible invariants formed from two derivatives and four Higgses, $\mathcal{O}\left(D^{2} H^{4}\right)$, we would find three terms (the 2 in the third term is for the 2 independent ways of contracting the $\mathrm{SU}(2)_{w}$ indices):

$$
\left(D_{\mu} H^{\dagger}\right)\left(D^{\mu} H^{\dagger}\right) H^{2}, \quad\left(D_{\mu} H\right)\left(D^{\mu} H\right) H^{\dagger 2}, \quad 2\left(D_{\mu} H^{\dagger}\right)\left(D^{\mu} H\right) H^{\dagger} H .
$$

However, these terms are not independent since IBP on either of the first two terms generates the third term, a total derivative, and a piece containing $\square H$ or $\square H^{\dagger}$. Therefore only the third term needs to be included in the operator counting. As a second example, consider the term at $\mathcal{O}\left(D^{4} H^{2}\right)$ :

$$
D_{\{\mu, \nu\}}^{2} H^{\dagger} D^{2,\{\mu \nu\}} H
$$

where the braces indicate the symmetric combination of indices $\left(D_{\{\mu, \nu\}}^{2}=\left\{D_{\mu}, D_{\nu}\right\}\right)$. Under IBP, this term only generates a total derivative and EOM terms, thus it completely vanishes from the physical operator basis.

Recently, a method to incorporate derivatives and their redundancies was pointed out in ref. [2] in the context of Hilbert series for scalar fields in $(0+1)$ dimensions. The starting point of ref. [2] was to dress each scalar field 'flavor' $\phi_{i}$ with a series of derivatives, $\phi_{i} \rightarrow \phi_{i}\left(1+\partial_{t}+\partial_{t}^{2}+\cdots\right)$. Because of the low dimensionality, the full series of both fields and derivatives could be summed, generating expressions for invariants to all orders in $\phi_{i}$ and $\partial_{t}$. While compact, this all-orders expression was initially plagued by the same IBP and EOM redundancies as our Hilbert series in four spacetime dimensions. However, ref. [2] showed these redundancies could be removed. The key to removing the redundancies was to write the Hilbert series as a nested sum, each power of $\phi_{i}$ weighted by a sum over derivatives. The derivative portion is a polynomial in the momenta, the number of momenta depending on the power of $\phi_{i}$. Each term in the derivative polynomial can be massaged and the pieces that represent EOM or IBP redundancies can be removed. For example, total momentum conservation could be used to swap out combinations of momenta, and any term in the polynomial with multiple powers of the same momentum $p_{i}^{2}$ - the Fourier transform of the EOM term $\partial_{t}^{2} \phi_{i}$ - could be removed. These constraints on the momentum polynomial can be elegantly phrased in the algebraic language of rings and ideals. After systematically

\footnotetext{
${ }^{4}$ Specifically, pieces proportional to the EOM do not contribute to on-shell matrix elements [50-54].
} 
cleaning up the momentum sum for each power of $\phi_{i}$, then summing over the powers of $\phi_{i}$, the resulting Hilbert series is free of all redundancies. For multiple flavors, the procedure is similar, though the nested sums become more difficult to work with. ${ }^{5}$

In higher spacetime dimensions, the Lorentz group is non-trivial, thus the transformation properties of derivatives become complicated and we cannot easily sum over all derivatives. However, our goals are also different; the aim of ref. [2] was the all-orders Hilbert series, while, at least for this work, we are interested in the subset of all operators at a specified mass dimension.

As we will now show, we can adapt several ideas from the $(0+1)$ dimensional method to four spacetime dimensions. We will first present the general algorithm, explain and motivate its ingredients, and show its limitations. Along the way we'll give several examples.

\section{Including derivatives in Hilbert series}

The algorithm we have constructed to include derivatives into Hilbert series in four spacetime dimensions and deal with their redundancies can be compactly written as the difference of two Hilbert series, with the coefficient of each term restricted to be positive:

$$
\max \left\{\mathfrak{H}_{\mathrm{EFT}}^{\text {single }}-D \mathfrak{H}_{D, \mathrm{EFT}}^{\text {single }}, 0\right\}
$$

Dropping the superscript for the moment, the first term is the Hilbert series for the EFT of interest with each spurion of the theory dressed with derivatives [1]. For a single real scalar field, dressing with derivatives means we modify the PE to

$$
\mathrm{PE}\left[\phi\left(1+D\left(\frac{1}{2}, \frac{1}{2}\right)+D^{2}(1,1)+D^{3}\left(\frac{3}{2}, \frac{3}{2}\right)+\cdots\right)\right] .
$$

Here, $D$ is a spurion for the derivative, and the numbers in parenthesis label the representation under $\mathrm{SU}(2)_{R} \otimes \mathrm{SU}(2)_{L} \cdot{ }^{6}$ Integrated over the Haar measure, this PE generates all invariant operators formed from $\phi, D_{\mu} \phi, D_{\{\mu, \nu\}}^{2} \phi$, etc. At order $D^{2}$ and higher, there are multiple ways to contract Lorentz indices, but we have only included the fully symmetric representation in eq. (2.2). The other possibilities are omitted because they are redundant by the EOM. For example, at $D^{2}$ there are two possible terms: $D_{\{\mu, \nu\}}^{2} \phi$ and $\square \phi$. However, in any invariant containing a $\square \phi$, the $\square \phi$ can be rewritten using the equations of motion as a term with fewer derivatives and is therefore already included in the PE (i.e. $\phi^{2} \square \phi$ reduces to $\phi^{3}$ ). At higher derivative orders, only the fully symmetric product of derivatives represents a genuinely new spurion and merits addition into the PE - all other combinations reduce through the EOM and are therefore redundant with terms that already exist. This process of removing the $\square$ terms is similar in spirit to projecting out the $\partial_{t}^{n}, n \geq 2$ pieces of the derivative polynomial in [2], though done here at the level of the argument of the PE.

\footnotetext{
${ }^{5}$ To get results for larger numbers of flavors, ref. [2] exploited an underlying $\operatorname{SL}(2, \mathbb{C})$ structure in the Hilbert series, where $\phi_{i}$ and its first derivative $\partial_{t} \phi_{i}$ form a doublet.

${ }^{6}$ Here, we use 1 for the trivial representation $(0,0)$.
} 
Similar logic follows for fermions [1]. Consider the EOM for the left-handed Standard Model quark doublet $Q$ :

$$
i \not D Q=y_{u}^{\dagger} u^{c \dagger} \epsilon H^{*}+y_{d}^{\dagger} d^{c \dagger} H
$$

which tells us that $\not D Q$ can be removed in favor of a combination of fields with no derivatives. However, $\not D Q$ is just one way to contract the Lorentz indices. Acting on a left-handed fermion with a derivative, we get (ignoring all non-Lorentz information): $\left(\frac{1}{2}, \frac{1}{2}\right) \otimes\left(0, \frac{1}{2}\right)=\left(\frac{1}{2}, 0\right) \oplus\left(\frac{1}{2}, 1\right)$. The EOM in eq. (2.3) involves the $\left(\frac{1}{2}, 0\right)$ representation only. Following the same prescription we used for our toy scalar theory, we can incorporate the EOM for a fermion field $Q$ (for example) by including an additional spurion for the $\left(\frac{1}{2}, 1\right)$ part of $D_{\mu} Q$, but omitting the $\left(\frac{1}{2}, 0\right)$ spurion. As with scalars, only the completely symmetric combinations of higher derivative terms generate new invariants, i.e. $D_{\{\mu \nu\}}^{2} Q \sim\left(1, \frac{3}{2}\right)$. Analogously, for derivatives of the field strength tensors $D_{\lambda} X_{\mu \nu}^{ \pm}$, the $\left(\frac{1}{2}, \frac{1}{2}\right)$ representation should be omitted, while the symmetric combination is kept (the $\left(\frac{1}{2}, \frac{3}{2}\right)$ or $\left.\left(\frac{3}{2}, \frac{1}{2}\right)\right)$.

One may worry that once we consider a gauge theory, the ordinary derivatives all become covariant derivatives (we have been sloppy in the above discussion about distinguishing between the two) and it is no longer true that derivatives commute. This is not an issue; $\left[D_{\mu}, D_{\nu}\right]$ has the same quantum numbers as $X_{\mu \nu}^{L, R}$, so the antisymmetric combinations of derivatives acting on a field are redundant with the field strength times that field [45]. Combined with the redundancy pointed out above, we can summarize the action of two derivatives on the fermion field $Q$ :

$$
D^{2} \times Q=D_{\{\mu \nu\}}^{2} Q+X_{\mu \nu}^{L} Q+X_{\mu \nu}^{R} Q+\square Q,
$$

where $X_{\mu \nu}^{L, R}$ stands for the field strength of any of the SM gauge groups. The last three terms are already present in the $\mathrm{PE}$ with fewer derivatives and therefore are redundant, so only $D_{\{\mu \nu\}}^{2} Q$ needs to be added. Higher derivatives and fields with other spins work the same way.

We will call the combination of the derivative-dressed PE for each spurion (scalars $\phi$, fermions $\psi$, or gauge fields $X$ ) $\mathrm{PE}_{\mathrm{EFT}}[\phi, \psi, X]$. One important feature of $\mathrm{PE}_{\mathrm{EFT}}$ above is that the same spurion $D$ appears wherever there is a derivative, i.e. $D \phi$ is really the product of two separate spurions and $D \phi$ and $D \psi$ involve the same $D$. This will be important for keeping track of the total number of derivatives in an invariant, which we need to do to in order to resolve the integration by parts redundancy. Another important feature is that the derivative dressing for each given spurion is technically an infinite series. While it is interesting to think about this infinite sum of derivatives, and we present some thoughts in appendix C, we will see shortly that eq. (2.1) fails at high derivative order so for our purposes we only need to keep $\mathcal{O}(D)$ and $\mathcal{O}\left(D^{2}\right)$.

Returning to eq. (2.1), we now discuss the second term $\mathfrak{H}_{D \text {, EFT }}$. This term contains all terms generated by the dressed PE that lie in the $\left(\frac{1}{2}, \frac{1}{2}\right)$ (four-vector) representation of the Lorentz group but are otherwise invariant

$$
\mathfrak{H}_{D, \mathrm{EFT}}=\int d \mu_{\mathrm{SU}(2)_{L}} d \mu_{\mathrm{SU}(2)_{R}}\left(\frac{1}{2}, \frac{1}{2}\right) \operatorname{PE}_{\mathrm{EFT}}[\phi, \psi, X] .
$$


Some examples are $\phi D_{\mu} \phi, D_{\{\mu, \nu\}}^{2} \phi D^{\nu} \phi \phi^{2}$, etc. We project out this subset using the same character orthogonality as in eq. (1.7). The argument of the integration is the same, $\mathrm{PE}_{\mathrm{EFT}}$; the only change is that we include a factor of the $\left(\frac{1}{2}, \frac{1}{2}\right)$ character function so that the objects in the four-vector representation are projected out.

Having identified $\mathfrak{H}_{\mathrm{EFT}}$ and $\mathfrak{H}_{D, \text { EFT }}$, the logic behind subtracting the two in eq. (2.1) is that any term with $2 n$ derivatives can be formed by acting on a term with $2 n-1$ derivatives with one additional derivative. Schematically, for scalar fields:

$$
\mathcal{O}\left(D^{2 n} \phi^{m}\right)=D \times \mathcal{O}\left(D^{2 n-1} \phi^{m}\right)
$$

with higher spin fields behaving similarly. Now, lets assume that there are $j$ operators at $O\left(D^{2 n} \phi^{m}\right)$ (taking scalars for simplicity) and $k$ operators at $\mathcal{O}\left(D^{2 n-1} \phi^{m}\right)$. As all of the $k$ $\mathcal{O}\left(D^{2 n} \phi^{m}\right)$ terms must be generated from the same $\mathcal{O}\left(D^{2 n-1} \phi^{m}\right)$ set with $j$ elements, the number of independent $\mathcal{O}\left(D^{2 n} \phi^{m}\right)$ is the difference:

$$
\text { (\# invariants })-(\text { \# relations })=k-j \text {. }
$$

Any terms that can be connected through a total derivative are removed by this subtraction, thus we are left with the number of invariants after taking integration by parts redundancies into account. The extra power of the spurion $D$ accompanying the $\mathfrak{H}_{D}$, EFT is necessary to ensure that the dimension and derivative counting matches in the two terms.

For low numbers of derivatives, this subtraction works perfectly. For example, lets apply this algorithm to a theory of a single scalar field at $\mathcal{O}\left(D^{2} \phi^{m}\right)$. There is only one term in the Hilbert series $\mathfrak{H}_{\phi}$ at $\mathcal{O}\left(D^{2} \phi^{m}\right)$, namely $\left(D_{\mu} \phi\right)\left(D^{\mu} \phi\right) \phi^{m-2}$. However

$$
\left(D_{\mu} \phi\right)\left(D^{\mu} \phi\right) \phi^{m-2} \sim\left(D_{\mu} \phi\right) D^{\mu}\left(\phi^{m-1}\right) \underset{\mathrm{IBP}}{\longrightarrow} \phi^{m-1} \square \phi \rightarrow \mathrm{EOM}
$$

so all $\mathcal{O}\left(D^{2} \phi^{m}\right)$ terms are actually redundant, via EOM and dropping total derivatives, with terms that have fewer derivatives (and are already in the PE). ${ }^{7}$ There is only one $\mathcal{O}\left(D \phi^{m}\right)$ term, namely $\left(D_{\mu} \phi\right) \phi^{m-1}$, generated by $\mathfrak{H}_{D \phi}$, so the prescription of eq. (2.1) for the improved Hilbert series correctly gives 0 terms.

Terms at $\mathcal{O}\left(D^{4} \phi^{m}\right)$ are also correctly counted by this method, however things go awry when we go to even higher derivatives. Focusing on terms with four $\phi$ fields, one can show that there are 6 operators at $\mathcal{O}\left(D^{6} \phi^{4}\right)$ and 6 at $\mathcal{O}\left(D^{5} \phi^{4}\right)$. This would indicate that no invariants are left after IBP. However, if we more closely examine the relations generated by the $6 \mathcal{O}\left(D^{5} \phi^{4}\right)$ operators, we see that only 5 are linearly independent. The simplest way to see this is to cast $D_{\mu} \mathcal{O}\left(D^{5} \phi^{4}\right)=0$ as a matrix times a vector of the $\mathcal{O}\left(D^{6} \phi^{4}\right)$ invariants, then determine the rank of the matrix. Subtracting the number of independent constraints from the number of operators, we find one operator at $\mathcal{O}\left(D^{6} \phi^{4}\right)$, consistent with direct computation. The same thing happens at $\mathcal{O}\left(D^{8} \phi^{4}\right)$; there are 11 invariants at $\mathcal{O}\left(D^{6} \phi^{4}\right)$, and 15 at $\mathcal{O}\left(D^{7} \phi^{4}\right)$, though only 10 of the 15 resulting relations are linearly independent.

Comparing these scalar examples, we see that the subtraction of eq. (2.1) correctly removes IBP redundancy only when the constraint equations (i.e $D \times \mathcal{O}\left(D^{2 n-1} \phi^{4}\right)=0$ for

\footnotetext{
${ }^{7}$ Throughout this paper, we are only concerned with the number of operators. The coefficient (including sign) in front of the operator is unimportant and will be neglected.
} 
the case of 4 fields) are linearly independent. The independence of the constraint equations is linked to the number of ways to partition the derivatives among the fields and the number of Lorentz invariants per partition. Using a notation $D^{i} \phi D^{j} \phi D^{k} \phi D^{m} \phi=(i, j, k, m)$ and ranking powers of $D$ in descending order, we can translate any given field content (number of derivatives $D$ and number of fields $\phi$ ) into a set of integer partitions representing different operators that can be formed from that field content: $D^{4} \phi^{4} \rightarrow(2,2,0,0),(2,1,1,0)$, $(1,1,1,1) ; D^{6} \phi^{4} \rightarrow(3,3,0,0),(3,2,1,0),(3,1,1,1),(2,2,2,0),(2,2,1,1)$, etc. For a some partitions, such as $(2,2,0,0)$, there is only one way to contract Lorentz indices, namely $D_{\{\mu, \nu\}} \phi D^{\{\mu, \nu\}} \phi \phi^{2}$; however at higher orders there are sometimes multiple possibilities. For example, the partition $(2,2,1,1)$ for $D^{6} \phi^{4}$ has two Lorentz contractions:

$$
D_{\{\mu, \nu\}}^{2} \phi D^{2,\{\mu, \nu\}} \phi D_{\rho} \phi D^{\rho} \phi \quad \text { and } \quad D_{\{\mu, \nu\}}^{2} \phi D^{2,\{\nu, \rho\}} \phi D^{\mu} \phi D_{\rho} \phi .
$$

If there is only one contraction per partition, eq. (2.1) simply counts the number of partitions of $2 n$ derivatives among $m$ fields minus the number of partitions of $2 n-1$ derivatives on the same number of fields. Each of the partitions of $2 n-1$ derivatives gives a single independent constraint equation. For example, applying a derivative to a term $\mathcal{O}\left(\phi^{n+4}\right)$ where four of the $\phi$ fields carry at least one derivative each yields the partitions:

$$
\begin{aligned}
D \otimes\left(D^{i} \phi D^{j} \phi D^{k} \phi D^{m} \phi \phi^{n}\right)= & (i+1, j, k, m, 0, \cdots)+(i, j+1, k, m, 0, \cdots)+ \\
& (i, j, k+1, m, 0, \cdots)+(i, j, k, m+1,0, \cdots)+ \\
& n(i, j, k, m, 1,0, \cdots) .
\end{aligned}
$$

If there is only one contraction per partition, then since the partitions are independent, so are the resulting constraint equations. On the other hand, if multiple contractions are possible, a given $2 n-1$ derivative partition may yield multiple constraint equations and the independence of the constraints breaks down. ${ }^{8}$

Clearly, one would like an implementation of derivatives in Hilbert series that can overcome the complication of multiple Lorentz contractions. It is possible to modify the subtraction by adding more terms, such that eq. (2.1) is correct for certain higher order terms, however we have not found a modification that works consistently for multiple types of fields or corrects all higher order terms. The pursuit of the full implementation of derivatives is enticing and an active area of research (and it is possible that the full implementation of derivatives will look nothing like this subtraction), but beyond the scope of this paper. Instead, we will carry on with eq. (2.1), adding the superscript "single" to both pieces to indicate that eq. (2.1) only seems to apply when there is a single Lorentz contraction per derivative partition. While restricted, eq. (2.1) is still sufficient to capture a large set of low-derivative operators. To get a better idea of where eq. (2.1) works and

\footnotetext{
${ }^{8}$ As further proof that the existence of multiple Lorentz contractions per derivative partition is what spoils the algorithm, we can apply eq. (2.1) to a $0+1$ dimensional theory. In the language we have been working with, the Hilbert series is $\mathrm{PE}[\phi(1+D)]$ (for a single field), and the analog of eq. (2.1) is $\max \{(1-D) \mathrm{PE}[\phi(1+D)], 0\}$. There are no Lorentz contractions to worry about in $0+1$ dimensions, and $\max \{(1-D) \mathrm{PE}[\phi(1+D)], 0\}$ correctly captures all operators of the Hilbert series as presented in eq. (3.9)-(3.12) of ref. [2].
} 
where it fails, we work through some examples. However, before proceeding, we emphasize that the Hilbert series of the derivative-dressed PE ( $\mathfrak{H}_{\mathrm{EFT}}$ in eq. (2.1)) does generate the full set of invariants of fields and their derivatives while correctly accounting for EOM redundancy. As such, even in cases where eq. (2.1) fails, $\mathfrak{H}_{\text {EFT }}$ alone will give an upper bound on the number of operators at a given mass dimension. Furthermore, one can always take the output of $\mathfrak{H}_{D \text {,EFT }}$ for a given set of fields, apply another derivative and (albeit, tediously) compute the rank of the resulting constraint matrix by hand.

\subsection{Example: complex scalar}

For a single real scalar field we saw that eq. (2.1) becomes untrustworthy once we go to $>6$ derivatives and $>4$ fields, or operators of dimension ten or higher. Moving to a theory of a single complex scalar field, all invariant operators must have the field content $D^{2 n} \phi^{m} \phi^{* m}$. Consider the operators with $m=2$. As there are two distinct fields, we have to adapt the partition notation to $\left(D^{i} \phi D^{j} \phi D^{k} \phi^{*} D^{m} \phi^{*}\right) \equiv(i, j ; k, m)$, where numbers of derivatives acting on $\phi$ and $\phi^{*}$ are separately listed in descending order and the division between $\phi$ and $\phi^{*}$ is indicated by the semicolon. This notation generalizes straightforwardly when there are even more fields present, i.e. $D^{i} \phi_{1} D^{j} \phi_{2} D^{k} \phi_{3} \phi_{3}=(i ; j ; k, 0)$.

At $\mathcal{O}\left(D^{2}\right)$ there are three partitions: $(1,0 ; 1,0),(1,1 ; 0,0)$ and $(0,0 ; 1,1)$; each with a single Lorentz contraction. At four derivatives, the 8 partitions are $(2,2 ; 0,0),(0,0 ; 2,2)$, $(2,1 ; 1,0),(1,0 ; 2,1),(2,0 ; 1,1),(1,1 ; 2,0),(2,0 ; 2,0)$ and $(1,1 ; 1,1)$. The last partition has two possible Lorentz contractions $\left(D_{\mu} \phi D^{\mu} \phi D_{\nu} \phi^{*} D^{\nu} \phi^{*}\right.$ and $\left.D_{\mu} \phi D^{\mu} \phi^{*} D_{\nu} \phi D^{\nu} \phi^{*}\right)$, so there are 9 operators before accounting for IBP. As expected, eq. (2.1) miscounts in this situation, predicting 1 invariant rather than the 2 independent invariants found the brute force way by calculating the rank of the constraint matrix $M$ (there are 8 constraint equations from IBP but $\operatorname{rank}(M)=7$ ). This example reinforces the notion that the subtraction in eq. (2.1) is limited by the presence of multiple Lorentz contractions and not at some fixed derivative order.

\subsection{Example: dimension-6 SMEFT}

Once we admit higher spin fields or internal symmetries, there are countless higher dimensional, high derivative operators one could look at to see whether they require multiple Lorentz contractions for a given derivative partitioning. Rather than march through possibilities, let us focus on operator types that appear in the SMEFT expansion, starting with dimension-6. While only a subset of all possibilities, the SMEFT operators will test how eq. (2.1) performs on a wide array of spin and gauge symmetry structures. We will roughly follow the formalism of ref. [45]: $\psi$ will stand for any fermion of either chirality, $X$ for any field strength ( $\mathrm{L}$ or $\mathrm{R}$ ), but we will use $H$ for a Higgs (both the field and its conjugate).

We only need to check eq. (2.1) against the SMEFT operators containing derivatives. Non-derivative terms will be correctly captured following the procedure in ref. [1] and recapped in the introduction.

At dimension 6, the highest number of derivatives that can appear is 4 : a term of $\mathcal{O}\left(D^{4} H^{\dagger} H\right)$. As there are two fields, there is only one partition of the derivatives, and only one Lorentz contraction for that partition. 
Continuing to lower numbers of derivatives following [45], we next have $\mathcal{O}\left(D^{3} \psi^{2}\right)$, where $\psi$ is either a left or right-handed fermion. Remembering that the derivative spurions in the PE for fermions are $D_{\mu} \psi_{L} \sim\left(\frac{1}{2}, 1\right), D_{\mu} \psi_{R} \sim\left(1, \frac{1}{2}\right)$, we can see that there is no way to partition the three derivatives among any pair of fermions that yields a Lorentz invariant, regardless of their chirality (even forgetting other quantum numbers). In other words, this term is redundant by EOM alone and would not even be generated by the derivative dressed SMEFT PE. ${ }^{9}$

At two derivatives there are four possible classes: $\mathcal{O}\left(D^{2} \psi^{2} H\right), \mathcal{O}\left(D^{2} \phi^{2} X\right), \mathcal{O}\left(D^{2} X^{2}\right)$, and $\mathcal{O}\left(D^{2} H^{2} H^{2 \dagger}\right)$.

- $\mathcal{O}\left(D^{2} \psi^{2} H\right)$. In this class, invariants can only be formed if both fermions have the same chirality and either both of the $\psi$ (which need not be the same) are acted on by a derivative, or one derivative acts on $\psi$ and one on $H$. In terms of partitions $D^{i} \psi_{a} ; D^{j} \psi_{b} ; D^{k} H=(i ; j ; k)$, these possibilities are $(1 ; 1 ; 0),(1 ; 0 ; 1)$ and $(0 ; 1 ; 1)$, and in all cases there is only Lorentz one contraction per partition. ${ }^{10}$

- $\mathcal{O}\left(D^{2} H^{\dagger} H X\right)$. Following our derivative dressing, derivatives of field strengths are only generated by the PE in (larger) Lorentz representations which have no way of forming singlet, so we only have to consider derivatives on the scalars. There is only one partition, $(1 ; 1 ; 0)$ in an obvious extension of the partition language we've been using, and only one contraction for that partition.

- $\mathcal{O}\left(D^{2} X^{2}\right)$. For $\left(X^{L}\right)^{2},\left(X^{R}\right)^{2}$ there is a single invariant partition $(1,1)$, with one contraction. For $X^{L} X^{R}$ there are no Lorentz invariants.

- $\mathcal{O}\left(D^{2} H^{2} H^{2 \dagger}\right)$. Both derivatives cannot act on a single field and yield a Lorentz invariant, but all other partitions are allowed: $(1,1 ; 0,0),(0,0 ; 1,1)$, and $(1,0 ; 1,0)$. For the first two, there is only one Lorentz contraction and only one $\mathrm{SU}(2)_{w}$ contraction; Bose symmetry forces the pairs of spurions, both the pair with derivatives and the pair without, to sit in the triplet representation of $\mathrm{SU}(2)_{w}$. The last partition also has only one Lorentz contraction, but has two $\mathrm{SU}(2)_{w}$ contractions since no two fields in the operator are identical.

Finally, the two classes with one derivative are $\mathcal{O}\left(D \psi^{2} X\right)$ and $\mathcal{O}\left(D \psi^{2} H^{\dagger} H\right)$.

- $\mathcal{O}\left(D \psi^{2} X\right)$. To be Lorentz invariant, the derivative must act on one of the fermions, and both fermions must have the same chirality. The partitions are $(1 ; 0 ; 0)$ (and $(0 ; 1 ; 0)$ if the $\psi$ represent two different fields). One one contraction is possible.

- $\mathcal{O}\left(D \psi^{2} H^{\dagger} H\right)$. The derivative must act on one of the scalars, so the allowed partitions are $(0 ; 0 ; 1 ; 0)$ and $(0 ; 0 ; 0 ; 1)$. Depending on the $\mathrm{SU}(2)_{w}$ quantum numbers of the fermions (which must have opposite chirality), there may be more than one $\mathrm{SU}(2)_{w}$ invariant, but each partition only allows a single Lorentz invariant.

\footnotetext{
${ }^{9}$ Terms of $\mathcal{O}\left(D^{4} X\right)$ do not appear for the same reason.

${ }^{10} \mathrm{As}$ listed, the partitions are for three different fields, i.e $e_{c} L H$. If both fermions were the same a moot case for the SMEFT given $\mathrm{SU}(2)_{w}$ and $\mathrm{U}(1)_{Y}$ quantum numbers - we would indicate partitions without the first semicolon.
} 
Having exhausted the list of dimension-6 SMEFT operators, we find that all operators have the form amenable to eq. (2.1), namely that each partition of derivatives (if any) among the fields in an operator only admits a single Lorentz contraction. Notice we haven't yet tested for the number of actual invariants for a given operator class; this will be done by eq. (2.1). So far we have simply tested whether these classes of operators fit the restrictions on whether eq. (2.1) can be used. To apply eq. (2.1), we form the derivative-dressed SM PE:

$$
\begin{aligned}
\mathrm{PE}_{\mathrm{tot}}^{\mathrm{SM}}= & \operatorname{PE}\left[H\left(0,0 ; 0, \frac{1}{2}, \frac{1}{2}\right)+D H\left(\frac{1}{2}, \frac{1}{2} ; 0, \frac{1}{2}, \frac{1}{2}\right)+D^{2} H\left(1,1 ; 0, \frac{1}{2}, \frac{1}{2}\right)+\cdots\right. \\
& \left.+B^{R}(1,0 ; 0,0,0)+D B^{R}\left(\frac{3}{2}, \frac{1}{2} ; 0,0,0\right)+\cdots+B^{R} \rightarrow W^{R}, G^{R}+\text { c.c. }\right] \times \\
& \operatorname{PEF}\left[Q\left(0, \frac{1}{2} ; 3,2, \frac{1}{6}\right)+D Q\left(\frac{1}{2}, 1 ; 3,2, \frac{1}{6}\right)+\cdots+Q \rightarrow u_{c}, d_{c}, L, e_{c}+\text { c.c. }\right],
\end{aligned}
$$

Here the numbers in parenthesis indicate the representation/quantum numbers under $\left(\mathrm{SU}(2)_{R}, \mathrm{SU}(2)_{L} ; \mathrm{SU}(3)_{c}, \mathrm{SU}(2)_{w}, \mathrm{U}(1)_{Y}\right)$ respectively (not to be confused with a derivative partition). Also, to clarify, the notation c.c. means adding in a new spurion for the conjugate of each field. For example, we need to add the spurion $H^{\dagger}$ for the Higgs conjugate field, along with the appropriate quantum numbers. Likewise, for fermions we need to add a spurion $\psi^{\dagger}$ for each fermion field $\psi$. As we are only interested in operators with less than four derivatives we can truncate each field's derivative dressing at $\mathcal{O}\left(D^{2}\right)$. Plugging $\mathrm{PE}_{\mathrm{tot}}^{\mathrm{SM}}$ into eq. (2.1), and performing the Haar integration, we find the following derivative terms at mass dimension 6 .

$$
\begin{gathered}
D\left(d_{c}^{\dagger} d_{c} H^{\dagger} H\right), \quad D\left(e_{c}^{\dagger} e_{c} H^{\dagger} H\right), 2 D\left(L^{\dagger} L H^{\dagger} H\right), \quad D\left(u_{c}^{\dagger} u_{c} H^{\dagger} H\right), \\
2 D\left(Q^{\dagger} Q H^{\dagger} H\right), \quad D\left(H^{2} d_{c}^{\dagger} u_{c}\right)+\text { h.c., } 2 D^{2}\left(H^{\dagger} H\right)^{2} .
\end{gathered}
$$

Notice that the derivatives appear as just another spurion in the sequence in the Hilbert series output. No information on where the derivative acts is provided, and this has to be figured out just like the other indices (see appendix B).

This set of derivative terms matches exactly with ref. [45]. Combined with the zero derivative, baryon number conserving terms that we already know will match from the analysis in [1], we reproduce the full set of 59 dimension- 6 operators. ${ }^{11}$ With one flavor of fermions, we find 4 baryon-number violating operators, all with zero derivatives. Adding these to the previous set gives a total of 63 operators, matching the total given in [45] aside from a single baryon-number-violating operator that vanishes in the single-flavor $\left(N_{f}=1\right)$ case that we are considering.

As a further check, we can repeat the calculation including multiple families of fermions, sending $Q \rightarrow N_{f} Q$, etc. in the full $\mathrm{PE}_{\text {tot }}^{\mathrm{SM}}$ (eq. (2.10)). The total number of operator coefficients for the baryon-number-conserving dimension-6 operators for various $N_{f}$ have been calculated previously in ref. [25], and we find these numbers are reproduced exactly by eq. (2.1) (specifically 76 coefficients for $N_{f}=1$ and 2499 coefficients for $N_{f}=3$ ).

\footnotetext{
${ }^{11}$ Since we use $X^{L}, X^{R}$ for gauge field strengths, our purely bosonic operators are not self-conjugate, while they are in the notation of [45]. To compare with [45], we need to include Hermitian conjugates for all purely boson operators.
} 
The success of eq. (2.1) in reproducing the dimension 6 SMEFT teaches us several things. First, it provides us with several examples that eq. (2.1) applies to operators containing fermions and field strengths as well, provided they lie within the restricted class of operators with a single Lorentz contraction per derivative partition. Second, we see that adding global or gauge symmetry to the theory does not immediately disrupt the subtraction setup - multiple internal symmetry contractions on an operator do not invalidate it from eq. (2.1), only multiple Lorentz contractions. Finally, we see that eq. (2.1) removes IBP redundancies even when operators contain fields with different gauge transformation properties. One may have worried that the gauge field pieces that are present in, i.e $D_{\mu} Q$ but not $D_{\mu} H$ (or, even more generally pieces in $D_{\mu}$ but not $\partial_{\mu}$ ) get subtracted off. However, the terms in $\mathfrak{H}_{D, \mathrm{EFT}}$ are only terms in the $(1 / 2,1 / 2)$ representation of the Lorentz group and trivial representation of all other groups: $(1 / 2,1 / 2 ; 0 \cdots)$, where the dots indicate no charge under any other quantum numbers, either local or global. Whenever $D_{\mu}$ contains non-abelian interactions, the non-abelian gauge field pieces sit in the adjoint representation of their respective gauge group (in addition to their Lorentz group representation) and not in $(1 / 2,1 / 2 ; 0 \cdots)$ alone. In other words, the subtraction in $\mathfrak{H}_{D, \mathrm{EFT}}$ appears to remove redundancies from shifting the $\partial_{\mu}$ piece among fields, with the extra pieces required to covariantize (or un-covariantize) the derivative simply dragged along to maintain gauge invariance.

The set of dimension- 6 operators given in [45] is not a unique representation. Operators can be related to combinations of other operators through the equations of motion, as we have seen, and also through general field redefinitions. While the number of parameters needed to describe the effective field theory at a given mass dimension cannot change, by picking a particular set, be it the set in refs. [34-36, 45, 55], we are choosing an operator basis. ${ }^{12}$ We have landed in the basis used by [45] due to our treatment of the equations of motion, whereby we removed terms whenever possible in favor of operators with fewer derivatives. While the choice of basis cannot matter when calculating a physical quantity (provided one works with a complete basis), picking the appropriate basis can certainly make a calculation simpler or make the mapping from a UV theory to the set of effective operators easier $[32,34,36]$.

\subsection{Example: dimension-7 SMEFT}

A further cross-check on eq. (2.1) is provided by using it to calculate the dimension-7 SMEFT operators. Doing this calculation for $N_{f}=1$ results in the following 6 operators containing one and two derivatives (all plus h.c.):

$$
D\left(e_{c}^{\dagger} d_{c}^{3}\right), \quad D\left(L e_{c}^{\dagger} H^{3}\right), \quad D\left(L Q^{\dagger} d_{c}^{2}\right), \quad D\left(L^{2} d_{c} u_{c}^{\dagger}\right), \quad 2\left(D^{2} L^{2} H^{2}\right)
$$

Combining these operators with the zero-derivative operators, we find 15 operators, matching the results of [47], up to operators that vanish in the flavor-diagonal $\left(N_{f}=1\right)$ case. ${ }^{13}$

\footnotetext{
${ }^{12} \mathrm{By}$ this we mean the basis for a fixed mass dimension.

${ }^{13} \mathrm{An}$ explicit calculation was done for $N_{f}=3 \mathrm{using}$ the Hilbert series, and these non-flavor-diagonal operators are indeed produced.
} 
This success seems to indicate that all operators containing derivatives at dimension- 7 admit only one Lorentz contraction per derivative partition. As this is non-trivial, we can go through the possible derivative operators in the same fashion we did for the dimension- 6 operators. Following ref. [47], and using the same notation as in the previous section, there are two possible operator classes with one derivative:

- $\mathcal{O}\left(D \psi^{2} H^{3}\right)$. To form a Lorentz invariant, the fermions must have opposite chirality and the derivative be applied to one of the Higgses. There may be multiple $\mathrm{SU}(2)_{w}$ contractions, but only one Lorentz contraction is possible.

- $\mathcal{O}\left(D \psi^{4}\right)$. A Lorentz singlet only arises when three fermions have one chirality, one of which carries the derivative, and the fourth fermion has the opposite chirality, i.e. $\psi_{R} D \psi_{L} \psi_{L}^{2}$. There may be several internal symmetry configurations that are permitted, or it may be the case that internal symmetry considerations only allow the derivative on a subset of the same-chirality fermions. In either case, there is only one Lorentz contraction.

At two derivatives, there is only a single class:

- $\mathcal{O}\left(D^{2} \psi^{2} H^{2}\right)$. Lorentz singlets can be formed if both $\psi$ (same chirality) are acted on by a derivative, both $H$, or one $\psi$ and one $H$ : partitions of $(1,1 ; 0,0),(0,0 ; 1,1)$, or $(1,0 ; 1,0)$ respectively. Partitions $(1,1 ; 0,0)$ and $(1,0 ; 1,0)$ are similar to cases considered earlier, and there is one Lorentz contraction in each case. The $(0,0 ; 1,1)$ partition is different as the Lorentz and $\mathrm{SU}(2)_{w}$ representations are linked by Bose/Fermi statistics. Specifically, the fermion pair, which must be a lepton doublet $L^{2}$ following the logic in [47] can either reside in the singlet $(0,0)$ or triplet $(0,1)$ representation of the Lorentz group, and each of those can be contracted with $(D H)^{2}$. However, the Lorentz singlet $L^{2}$ must be an $\mathrm{SU}(2)_{w}$ triplet, while the Lorentz triplet combination must be an $\mathrm{SU}(2)_{w}$ singlet. Thus, while there are multiple Lorentz contractions for the $(0,0 ; 1,1)$ derivative partition, there is only one contraction per partition for a given assignment of internal symmetry charges.

So, since eq. (2.1) correctly reproduces the dimension-7 operators (at least for $N_{f}=1$ ), we have learned that the internal quantum numbers can be important. This means that, when considering the SMEFT at higher mass dimension or when looking at EFT outside of the SM, we cannot determine the viability of eq. (2.1) based on the Lorentz properties of the field in the operators alone.

Finally, as the calculation of the number of dimension-7 operators for arbitrary $N_{f}$ has not yet been done in the literature, the results of this Hilbert series computation, based on assuming that eq. (2.1) is correct, are shown in appendix D. 


\section{SMEFT: dimension-8 operators}

We can now use the prescription of eq. (2.1) to study SMEFT dimension-8 operators for $N_{f}=1$. Generalizing from the previous examples, we conjecture that eq. (2.1) correctly subtracts off IBP redundancies only when there is a single Lorentz contraction for a given partition of derivatives and assignment of internal symmetry charges. As we will see, these restrictions prevent us from automatically generating the full set of dimension 8 SMEFT operators. However, we can generate a larger subset than has been shown previously, and set up a prescription for filling in the remaining operator classes.

To begin with, as no complete list of dimension-8 SMEFT operators without derivatives have been shown, we list the terms at $\mathcal{O}\left(D^{0}\right)$. These can be obtained following the recipe in the introduction using $\mathrm{PE}_{\mathrm{tot}}^{\mathrm{SM}}$ defined in eq. (2.10). We emphasize that this calculation of the derivative-free operators does not depend upon our conjectured algorithm for dealing with derivatives. Readers interested only in which derivative terms are allowed and which are not should skip to section 3.2.

\subsection{Derivative-free operators}

Following refs. [45, 47], we can group terms into classes governed by number of scalars, fermions, and field strengths. We will continue to use $H$ to represent Higgses (field or conjugate), $\psi$ for any fermion, and $X$ for any field strength. At dimension- 8 and no derivatives, there are 9 operator classes: $\mathcal{O}\left(H^{8}\right), \mathcal{O}\left(\psi^{2} H^{5}\right), \mathcal{O}\left(\psi^{4} H^{2}\right), \mathcal{O}\left(\psi^{4} X\right), \mathcal{O}\left(\psi^{2} H X^{2}\right)$, $\mathcal{O}\left(\psi^{2} H^{3} X\right), \mathcal{O}\left(H^{4} X^{2}\right), \mathcal{O}\left(H^{2} X^{3}\right)$ and $\mathcal{O}\left(X^{4}\right) \cdot{ }^{14}$

Class $\mathcal{O}\left(\boldsymbol{H}^{8}\right)$. At 8 Higgs fields (counting both $H$ and $H^{\dagger}$ as Higgses) there is only one term

$$
\left(H^{\dagger} H\right)^{4}
$$

Class $\mathcal{O}\left(\psi^{2} \boldsymbol{H}^{5}\right)$. This class contains 3 operators (all plus h.c.)

$$
\left(L e_{c} H^{\dagger}\right)\left(H^{\dagger} H\right)^{2}, \quad\left(Q d_{c} H^{\dagger}\right)\left(H^{\dagger} H\right)^{2}, \quad\left(Q u_{c} H\right)\left(H^{\dagger} H\right)^{2} .
$$

Class $\mathcal{O}\left(\boldsymbol{\psi}^{4} \boldsymbol{H}^{2}\right)$. We can divide this class further depending on the chirality of the fermions. There are 7 operators with two Higgses and only $\mathrm{SU}(2)_{w}$ singlet fermions:

$$
\begin{gathered}
\left(e_{c}^{\dagger} e_{c}\right)^{2}\left(H^{\dagger} H\right), \quad\left(d_{c}^{\dagger} d_{c}\right)^{2}\left(H^{\dagger} H\right), \quad\left(u_{c}^{\dagger} u_{c}\right)^{2}\left(H^{\dagger} H\right), \\
\left(d_{c}^{\dagger} d_{c}\right)\left(e_{c}^{\dagger} e_{c}\right)\left(H^{\dagger} H\right), \quad\left(u_{c}^{\dagger} u_{c}\right)\left(e_{c}^{\dagger} e_{c}\right)\left(H^{\dagger} H\right), 2\left(u_{c}^{\dagger} u_{c}\right)\left(d_{c}^{\dagger} d_{c}\right)\left(H^{\dagger} H\right),
\end{gathered}
$$

and 10 operators with only $\mathrm{SU}(2)_{w}$ doublet fermions. Both the all-doublet and all-singlet operators sets involve self-Hermitian operators

$$
2\left(L^{\dagger} L\right)^{2}\left(H^{\dagger} H\right), 3\left(Q^{\dagger} Q\right)^{2}\left(H^{\dagger} H\right), \quad 5\left(L^{\dagger} L\right)\left(Q^{\dagger} Q\right)\left(H^{\dagger} H\right)
$$

\footnotetext{
${ }^{14}$ While operators $\mathcal{O}\left(H^{6} X\right)$ have dimension 8 , there is no way to make a Lorentz invariant without derivatives, thus there are no operators of this type at $\mathcal{O}\left(D^{0}\right)$.
} 
Operators involving both $\mathrm{SU}(2)_{w}$ singlet and doublet fermions can be either self-Hermitian or not. There are 16 self-Hermitian operators:

$$
\begin{array}{ccc}
2\left(L^{\dagger} L\right)\left(d_{c}^{\dagger} d_{c}\right)\left(H^{\dagger} H\right), & 2\left(L^{\dagger} L\right)\left(u_{c}^{\dagger} u_{c}\right)\left(H^{\dagger} H\right), & 2\left(L^{\dagger} L\right)\left(e_{c}^{\dagger} e_{c}\right)\left(H^{\dagger} H\right), \\
2\left(Q^{\dagger} Q\right)\left(e_{c}^{\dagger} e_{c}\right)\left(H^{\dagger} H\right), & 4\left(Q^{\dagger} Q\right)\left(d_{c}^{\dagger} d_{c}\right)\left(H^{\dagger} H\right), & 4\left(Q^{\dagger} Q\right)\left(u_{c}^{\dagger} u_{c}\right)\left(H^{\dagger} H\right),
\end{array}
$$

and 21 operators with Hermitian conjugates:

$$
\begin{aligned}
& e_{c}^{2} L^{2}\left(H^{\dagger}\right)^{2}, 2 d_{c}^{2} Q^{2}\left(H^{\dagger}\right)^{2}, 2 u_{c}^{2} Q^{2} H^{2}, 2\left(d_{c} Q\right)\left(e_{c} L\right)\left(H^{\dagger}\right)^{2}, \\
& 2\left(d_{c} Q\right)\left(e_{c}^{\dagger} L^{\dagger}\right)\left(H^{\dagger} H\right), \quad\left(d_{c}^{\dagger} u_{c}\right)\left(L^{\dagger} L\right) H^{2}, 4\left(u_{c} Q\right)\left(e_{c} L\right)\left(H^{\dagger} H\right), \\
& \left(u_{c} Q\right)\left(e_{c}^{\dagger} L^{\dagger}\right) H^{2}, \quad 4\left(u_{c} Q\right)\left(d_{c} Q\right)\left(H^{\dagger} H\right), 2\left(u_{c} Q\right)\left(d_{c}^{\dagger} Q^{\dagger}\right) H^{2} \text {. }
\end{aligned}
$$

Finally, there are 6 baryon-number-violating operators (all plus h.c.):

$$
2 L Q^{3}\left(H^{\dagger} H\right), 2\left(u_{c} d_{c}\right)\left(Q^{\dagger} L^{\dagger}\right)\left(H^{\dagger} H\right), \quad\left(e_{c} u_{c}\right)\left(Q^{\dagger}\right)^{2}\left(H^{\dagger} H\right), \quad\left(e_{c} d_{c}\right) u_{c}^{2}\left(H^{\dagger} H\right)
$$

Class $\mathcal{O}\left(\psi^{4} \boldsymbol{X}\right)$. There are 78 operators with four fermions and a single field strength (all plus h.c.):

$$
\begin{gathered}
\left(d_{c}^{\dagger} d_{c}\right)\left(e_{c}^{\dagger} e_{c}\right) B^{L}, \quad\left(u_{c}^{\dagger} u_{c}\right)\left(e_{c}^{\dagger} e_{c}\right) B^{L}, \quad 2\left(d_{c}^{\dagger} d_{c}\right)\left(u_{c}^{\dagger} u_{c}\right) B^{L}, \quad\left(d_{c}^{\dagger} d_{c}\right)\left(L^{\dagger} L\right) B^{L}, \quad\left(u_{c}^{\dagger} u_{c}\right)\left(L^{\dagger} L\right) B^{L}, \\
\left(e_{c}^{\dagger} e_{c}\right)\left(L^{\dagger} L\right) B^{L}, \quad\left(e_{c}^{\dagger} e_{c}\right)\left(Q^{\dagger} Q\right) B^{L}, \quad\left(d_{c} Q\right)\left(e_{c}^{\dagger} L^{\dagger}\right) B^{L}, \quad\left(d_{c} Q\right)\left(e_{c}^{\dagger} L^{\dagger}\right) B^{R}, 2\left(L^{\dagger} L\right)\left(Q^{\dagger} Q\right) B^{L}, \\
2\left(d_{c}^{\dagger} d_{c}\right)\left(Q^{\dagger} Q\right) B^{L}, 2\left(u_{c}^{\dagger} u_{c}\right)\left(Q^{\dagger} Q\right) B^{L}, \quad 3\left(e_{c} L\right)\left(u_{c} Q\right) B^{L}, 3\left(u_{c} d_{c}\right) Q^{2} B^{L}, \quad\left(d_{c}^{\dagger} d_{c}\right)\left(L^{\dagger} L\right) W^{L}, \\
\left(e_{c}^{\dagger} e_{c}\right)\left(L^{\dagger} L\right) W^{L}, \quad\left(e_{c}^{\dagger} e_{c}\right)\left(Q^{\dagger} Q\right) W^{L}, \quad\left(u_{c}^{\dagger} u_{c}\right)\left(L^{\dagger} L\right) W^{L}, \quad\left(L^{\dagger} L\right)^{2} W^{L}, \quad\left(e_{c}^{\dagger} L^{\dagger}\right)\left(d_{c} Q\right) W^{L}, \\
\left(e_{c} L\right)\left(d_{c}^{\dagger} Q^{\dagger}\right) W^{L}, \quad 2\left(d_{c}^{\dagger} d_{c}\right)\left(Q^{\dagger} Q\right) W^{L}, 2\left(u_{c}^{\dagger} u_{c}\right)\left(Q^{\dagger} Q\right) W^{L}, \quad 3\left(L^{\dagger} L\right)\left(Q^{\dagger} Q\right) W^{L}, 2\left(Q^{\dagger} Q\right)^{2} W^{L}, \\
3\left(e_{c} L\right)\left(u_{c} Q\right) W^{L}, \quad 3\left(u_{c} d_{c}\right) Q^{2} W^{L}, \quad\left(d_{c}^{\dagger}\right)^{2} d_{c}^{2} G^{L}, \quad\left(u_{c}^{\dagger}\right)^{2} u_{c}^{2} G^{L}, \quad\left(d_{c}^{\dagger} d_{c}\right)\left(e_{c}^{\dagger} e_{c}\right) G^{L}, \\
\left(u_{c}^{\dagger} u_{c}\right)\left(e_{c}^{\dagger} e_{c}\right) G^{L}, \quad 4\left(d_{c}^{\dagger} d_{c}\right)\left(u_{c}^{\dagger} u_{c}\right) G^{L}, \quad\left(Q^{\dagger} Q\right)\left(e_{c}^{\dagger} e_{c}\right) G^{L}, \quad\left(d_{c}^{\dagger} d_{c}\right)\left(L^{\dagger} L\right) G^{L}, \quad\left(u_{c}^{\dagger} u_{c}\right)\left(L^{\dagger} L\right) G^{L}, \\
2\left(Q^{\dagger} Q\right)\left(L^{\dagger} L\right) G^{L}, \quad 4\left(d_{c}^{\dagger} d_{c}\right)\left(Q^{\dagger} Q\right) G^{L}, \quad 4\left(u_{c}^{\dagger} u_{c}\right)\left(Q^{\dagger} Q\right) G^{L}, 2\left(Q^{\dagger}\right)^{2} Q^{2} G^{L}, \quad\left(d_{c} Q\right)\left(e_{c}^{\dagger} L^{\dagger}\right) G^{L}, \\
\left(d_{c} Q\right)\left(e_{c}^{\dagger} L^{\dagger}\right) G^{R}, \quad 3\left(e_{c} L\right)\left(u_{c} Q\right) G^{L}, \quad 6\left(d_{c} u_{c}\right) Q^{2} G^{L} .
\end{gathered}
$$

Additionally, this class contains 22 baryon-number-violating operators (all plus h.c.):

$$
\begin{gathered}
L Q^{3} B^{L}, \quad\left(u_{c} d_{c}\right)\left(L^{\dagger} Q^{\dagger}\right) B^{L}, \quad\left(u_{c} d_{c}\right)\left(L^{\dagger} Q^{\dagger}\right) B^{R}, \quad\left(e_{c} u_{c}\right)\left(Q^{\dagger}\right)^{2} B^{L}, 2\left(e_{c} d_{c}\right) u_{c}^{2} B^{L}, \\
2 L Q^{3} W^{L}, \quad\left(u_{c} d_{c}\right)\left(L^{\dagger} Q^{\dagger}\right) W^{L}, \quad\left(u_{c} d_{c}\right)\left(L^{\dagger} Q^{\dagger}\right) W^{R}, \quad\left(e_{c}^{\dagger} u_{c}^{\dagger}\right) Q^{2} W^{L}, \\
2 L Q^{3} G^{L}, 2\left(u_{c} d_{c}\right)\left(L^{\dagger} Q^{\dagger}\right) G^{L}, 2\left(u_{c} d_{c}\right)\left(L^{\dagger} Q^{\dagger}\right) G^{R}, \\
\left(e_{c} u_{c}\right)\left(Q^{\dagger}\right)^{2} G^{L}, \quad\left(e_{c} u_{c}\right)\left(Q^{\dagger}\right)^{2} G^{R}, 3\left(e_{c} d_{c}\right) u_{c}^{2} G^{L} .
\end{gathered}
$$

Class $\mathcal{O}\left(\psi^{2} H^{3} \boldsymbol{X}\right)$. This class contains 11 operators (all plus h.c.),

$\left(e_{c} L\right) H^{\dagger}\left(H^{\dagger} H\right) B^{L}, \quad\left(d_{c} Q\right) H^{\dagger}\left(H^{\dagger} H\right) B^{L}, \quad\left(u_{c} Q\right) H\left(H^{\dagger} H\right) B^{L}, 2\left(e_{c} L\right) H^{\dagger}\left(H^{\dagger} H\right) W^{L}$, $2\left(d_{c} Q\right) H^{\dagger}\left(H^{\dagger} H\right) W^{L}, 2\left(u_{c} Q\right) H\left(H^{\dagger} H\right) W^{L}, \quad\left(d_{c} Q\right) H^{\dagger}\left(H^{\dagger} H\right) G^{L}, \quad\left(u_{c} Q\right) H\left(H^{\dagger} H\right) G^{L}$. 
Class $\mathcal{O}\left(\psi^{2} \boldsymbol{H} \boldsymbol{X}^{2}\right)$. The 48 operators (all plus h.c.) in this class are:

$$
\begin{gathered}
\left(e_{c} L\right) H^{\dagger}\left(B^{L}\right)^{2}, \quad\left(e_{c} L\right) H^{\dagger}\left(B^{R}\right)^{2}, \quad\left(d_{c} Q\right) H^{\dagger}\left(B^{L}\right)^{2}, \quad\left(d_{c} Q\right) H^{\dagger}\left(B^{R}\right)^{2}, \\
\left(u_{c} Q\right) H\left(B^{L}\right)^{2}, \quad\left(u_{c} Q\right) H\left(B^{R}\right)^{2}, \quad 2\left(e_{c} L\right) H^{\dagger} B^{L} W^{L}, \quad\left(e_{c}^{\dagger} L^{\dagger}\right) H B^{L} W^{L}, \\
2\left(d_{c} Q\right) H^{\dagger} B^{L} W^{L}, \quad\left(d_{c}^{\dagger} Q^{\dagger}\right) H B^{L} W^{L}, \quad 2\left(u_{c} Q\right) H B^{L} W^{L}, \quad\left(u_{c}^{\dagger} Q^{\dagger}\right) H^{\dagger} B^{L} W^{L}, \\
2\left(e_{c} L\right) H^{\dagger}\left(W^{L}\right)^{2}, \quad\left(e_{c}^{\dagger} L^{\dagger}\right) H\left(W^{L}\right)^{2}, \quad 2\left(d_{c} Q\right) H^{\dagger}\left(W^{L}\right)^{2}, \quad\left(d_{c}^{\dagger} Q^{\dagger}\right) H\left(W^{L}\right)^{2}, \\
2\left(u_{c} Q\right) H\left(W^{L}\right)^{2}, \quad\left(u_{c}^{\dagger} Q^{\dagger}\right) H^{\dagger}\left(W^{L}\right)^{2}, \quad 2\left(d_{c} Q\right) H^{\dagger} B^{L} G^{L}, \quad\left(d_{c} Q\right) H^{\dagger} B^{R} G^{R}, \\
2\left(d_{c} Q\right) H^{\dagger} W^{L} G^{L}, \quad\left(d_{c} Q\right) H^{\dagger} W^{R} G^{R}, \quad 2\left(u_{c} Q\right) H B^{L} G^{L}, \quad\left(u_{c} Q\right) H B^{R} G^{R}, \\
2\left(u_{c} Q\right) H W^{L} G^{L}, \quad\left(u_{c} Q\right) H W^{R} G^{R}, \quad\left(e_{c} L\right) H^{\dagger}\left(G^{L}\right)^{2}, \quad\left(e_{c} L\right) H^{\dagger}\left(G^{R}\right)^{2}, \\
3\left(d_{c} Q\right) H^{\dagger}\left(G^{L}\right)^{2}, \quad 2\left(d_{c} Q\right) H^{\dagger}\left(G^{R}\right)^{2}, \quad 3\left(u_{c} Q\right) H\left(G^{L}\right)^{2}, \quad 2\left(u_{c} Q\right) H\left(G^{R}\right)^{2} .
\end{gathered}
$$

Class $\mathcal{O}\left(\boldsymbol{H}^{4} \boldsymbol{X}^{2}\right)$. There are 5 operators (all plus h.c.) in this class:

$$
\left(H^{\dagger} H\right)^{2}\left(B^{L}\right)^{2}, \quad\left(H^{\dagger} H\right)^{2} B^{L} W^{L}, 2\left(H^{\dagger} H\right)^{2}\left(W^{L}\right)^{2}, \quad\left(H^{\dagger} H\right)^{2}\left(G^{L}\right)^{2} .
$$

Class $\mathcal{O}\left(\boldsymbol{H}^{2} \boldsymbol{X}^{\mathbf{3}}\right)$. Here we find 3 operators (all plus h.c.):

$$
\left(H^{\dagger} H\right) B^{L}\left(W^{L}\right)^{2}, \quad\left(H^{\dagger} H\right)\left(W^{L}\right)^{3}, \quad\left(H^{\dagger} H\right)\left(G^{L}\right)^{3},
$$

Class $\mathcal{O}\left(\boldsymbol{X}^{4}\right)$. The final class contains 9 self-Hermitian operators

$$
\begin{aligned}
& \left(B^{L}\right)^{2}\left(B^{R}\right)^{2}, \quad 2\left(W^{L}\right)^{2}\left(W^{R}\right)^{2}, \quad 3\left(G^{L}\right)^{2}\left(G^{R}\right)^{2}, \\
& B^{L} B^{R} W^{L} W^{R}, \quad B^{L} B^{R} G^{L} G^{R}, \quad G^{L} G^{R} W^{L} W^{R},
\end{aligned}
$$

and 17 operators with Hermitian conjugates

$$
\begin{gathered}
\left(B^{L}\right)^{4}, 2\left(W^{L}\right)^{4}, \quad 3\left(G^{L}\right)^{4}, \\
2\left(B^{L}\right)^{2}\left(W^{L}\right)^{2}, \quad\left(B^{R}\right)^{2}\left(W^{L}\right)^{2}, 2\left(B^{L}\right)^{2}\left(G^{L}\right)^{2}, \quad\left(B^{R}\right)^{2}\left(G^{L}\right)^{2}, \\
B^{L}\left(G^{L}\right)^{3}, \quad B^{R}\left(G^{L}\right)^{2} G^{R}, 2\left(W^{L}\right)^{2}\left(G^{L}\right)^{2}, \quad\left(W^{L}\right)^{2}\left(G^{R}\right)^{2} .
\end{gathered}
$$

All in all, for $N_{f}=1$ there are 257 dimension- 8 operators without derivatives (not counting Hermitian conjugates). The total comes to 471 operators if Hermitian conjugates are included. Of these 257 operators, 64 are without field strength tensors, and the remaining 193 contain at least one $X_{\mu \nu}$. The baryon-number-violating operators at $\mathcal{O}\left(D^{0}\right)$ make up 28 of the 257 operators.

\subsection{Operators with derivatives: $\mathcal{O}(D)$}

Moving to operators with derivatives, we now have to see which terms can we use with our simple subtraction algorithm eq. (2.1). We'll begin by enumerating the operator classes at each derivative order, then checking class by class. However, as we learned from dimension-7, sometimes we need more detail than just which fields (scalar, fermion, field strength) compose a particular class. 
At $\mathcal{O}(D)$ there are four classes: $\mathcal{O}\left(D \psi^{2} H^{4}\right), \mathcal{O}\left(D \psi^{4} H\right), \mathcal{O}\left(\psi^{2} X^{2}\right)$ and $\mathcal{O}\left(\psi^{2} H^{2} X\right)$

- $\mathcal{O}\left(D \psi^{2} H^{4}\right)$. This can be Lorentz invariant only if the derivative is applied to one of the Higgs fields and the fermions have opposite chirality, thus there is only one Lorentz contraction per partition regardless of internal quantum numbers.

- $\mathcal{O}\left(D \psi^{4} H\right)$. The fermion chiralities must be either LLLR or RRRL, and the derivative must act either on the Higgs field or one of the fermions of the dominant chirality. In either case, there is only one Lorentz contraction, though internal quantum numbers may forbid or augment certain derivative locations. ${ }^{15}$

- $\mathcal{O}\left(D \psi^{2} X^{2}\right)$. When the gauge fields are $X^{L} X^{R}$, the derivative must act on one of the fermions, and the two fermions must have opposite chirality. Each possibility has a single Lorentz contraction. When the field strengths are $\left(X^{L}\right)^{2}$ or $\left(X^{R}\right)^{2}$, the fermions still must have opposite chirality. The derivative can act on one of the gauge fields or on the LH fermion (for $\left(X^{L}\right)^{2}$ ) or RH fermion (for $\left(X^{R}\right)^{2}$ ), and both choices admit a single Lorentz contraction.

- $O\left(\psi^{2} H^{2} X\right)$. Operators in this class can only be Lorentz singlets if the fermions have opposite chirality and the derivative acts on one of the Higgses. There is only one contraction.

All $\mathcal{O}(D)$ operators at dimension-8 pass the criteria for eq. (2.1). Applying it using eq. (2.10) as the argument, the results are listed below and grouped by class.

Class $\mathcal{O}\left(\boldsymbol{D} \psi^{2} \boldsymbol{H}^{4}\right)$. Includes the 11 self-Hermitian operators

$$
\begin{gathered}
D\left(d_{c}^{\dagger} d_{c}\left(H^{\dagger} H\right)^{2}\right), \quad D\left(e_{c}^{\dagger} e_{c}\left(H^{\dagger} H\right)^{2}\right), \quad D\left(u_{c}^{\dagger} u_{c}\left(H^{\dagger} H\right)^{2}\right), \\
4 D\left(L^{\dagger} L\left(H^{\dagger} H\right)^{2}\right), 4 D\left(Q^{\dagger} Q\left(H^{\dagger} H\right)^{2}\right),
\end{gathered}
$$

and a single operator with a Hermitian conjugate

$$
D\left(d_{c}^{\dagger} u_{c} H^{3} H^{\dagger}\right)
$$

Class $\mathcal{O}\left(\boldsymbol{D} \boldsymbol{\psi}^{4} \boldsymbol{H}\right)$. This class contains 67 operators (all plus h.c.):

$$
\begin{aligned}
& 3 D\left(d_{c}^{\dagger} d_{c} L e_{c} H^{\dagger}\right), \quad D\left(e_{c}^{\dagger} e_{c} L e_{c} H^{\dagger}\right), 3 D\left(L^{\dagger} L^{2} e_{c} H^{\dagger}\right), 3 D\left(d_{c}^{\dagger} d_{c}^{2} Q H^{\dagger}\right), \\
& 3 D\left(e_{c}^{\dagger} e_{c} Q d_{c} H^{\dagger}\right), 6 D\left(L^{\dagger} L Q d_{c} H^{\dagger}\right), 6 D\left(Q^{\dagger} Q L e_{c} H^{\dagger}\right), 6 D\left(Q^{\dagger} Q^{2} d_{c} H^{\dagger}\right) \text {, } \\
& 3 D\left(d_{c}^{\dagger} u_{c} L e_{c} H\right), 6 D\left(d_{c}^{\dagger} d_{c} Q u_{c} H\right), 3 D\left(e_{c}^{\dagger} e_{c} Q u_{c} H\right), 6 D\left(L^{\dagger} L Q u_{c} H\right) \text {, } \\
& 6 D\left(Q^{\dagger} Q^{2} u_{c} H\right), 3 D\left(u_{c}^{\dagger} u_{c} L e_{c} H^{\dagger}\right), 6 D\left(u_{c}^{\dagger} u_{c} Q d_{c} H^{\dagger}\right), 3 D\left(u_{c}^{\dagger} u_{c}^{2} Q H\right) \text {. }
\end{aligned}
$$

as well as 16 baryon-number-violating operators (all plus h.c.):

$$
\begin{gathered}
3 D\left(d_{c}^{\dagger} Q^{2} L H\right), D\left(e_{c}^{\dagger} Q^{3} H\right), 2 D\left(d_{c}^{2} u_{c} L^{\dagger} H^{\dagger}\right), 3 D\left(d_{c} e_{c} Q^{\dagger} u_{c} H^{\dagger}\right), \\
3 D\left(\left(Q^{\dagger}\right)^{2} L^{\dagger} u_{c} H\right), 2 D\left(L^{\dagger} u_{c}^{2} d_{c} H\right), 2 D\left(Q^{\dagger} u_{c}^{2} e_{c} H\right) .
\end{gathered}
$$

\footnotetext{
${ }^{15}$ An example of with fewer derivative possibilities is $D\left(e_{c}^{\dagger} e_{c}^{2} L H\right)$, where two fermion fields are identical, and example of a combination with more is $D\left(L^{\dagger} L Q d_{c} H\right)$.
} 
Class $\mathcal{O}\left(D \psi^{2} \boldsymbol{X}^{\mathbf{2}}\right)$. This class contains 23 self-Hermitian operators:

$$
\begin{gathered}
D\left(e_{c}^{\dagger} e_{c} B^{L} B^{R}\right), \quad D\left(d_{c}^{\dagger} d_{c} B^{L} B^{R}\right), \quad D\left(u_{c}^{\dagger} u_{c} B^{L} B^{R}\right), \quad D\left(Q^{\dagger} Q B^{L} B^{R}\right), \\
D\left(L^{\dagger} L B^{L} B^{R}\right), \quad 3 D\left(d_{c}^{\dagger} d_{c} G^{L} G^{R}\right), \quad D\left(e_{c}^{\dagger} e_{c} G^{L} G^{R}\right), \quad D\left(L^{\dagger} L G^{L} G^{R}\right), \\
3 D\left(Q^{\dagger} Q G^{L} G^{R}\right), \quad 3 D\left(u_{c}^{\dagger} u_{c} G^{L} G^{R}\right), \quad D\left(d_{c}^{\dagger} d_{c} W^{L} W^{R}\right), \quad D\left(e_{c}^{\dagger} e_{c} W^{L} W^{R}\right), \\
D\left(u_{c}^{\dagger} u_{c} W^{L} W^{R}\right), \quad 2 D\left(L^{\dagger} L W^{L} W^{R}\right), \quad 2 D\left(Q^{\dagger} Q W^{L} W^{R}\right),
\end{gathered}
$$

and 17 operators with Hermitian conjugates

$$
\begin{array}{cccc}
D\left(d_{c}^{\dagger} d_{c} B^{L} G^{L}\right), & D\left(d_{c}^{\dagger} d_{c} B^{R} G^{L}\right), & D\left(d_{c}^{\dagger} d_{c}\left(G^{L}\right)^{2}\right), & D\left(Q^{\dagger} Q B^{L} G^{L}\right), \\
D\left(Q^{\dagger} Q B^{R} G^{L}\right), & D\left(Q^{\dagger} Q\left(G^{L}\right)^{2}\right), & D\left(u_{c}^{\dagger} u_{c} B^{L} G^{L}\right), & D\left(u_{c}^{\dagger} u_{c} B^{R} G^{L}\right), \\
D\left(u_{c}^{\dagger} u_{c}\left(G^{L}\right)^{2}\right), & D\left(L^{\dagger} L B^{L} W^{L}\right), & D\left(L^{\dagger} L B^{R} W^{L}\right), & D\left(Q^{\dagger} Q B^{L} W^{L},\right. \\
D\left(Q^{\dagger} Q B^{R} W^{L}\right), & D\left(Q^{\dagger} Q G^{L} W^{L}\right), & D\left(Q^{\dagger} Q G^{R} W^{L}\right), & D\left(L^{\dagger} L\left(W^{L}\right)^{2}\right), \\
D\left(Q^{\dagger} Q\left(W^{L}\right)^{2}\right) . &
\end{array}
$$

Class $\mathcal{O}\left(\boldsymbol{D} \psi^{2} \boldsymbol{H}^{2} \boldsymbol{X}\right)$. This last class contains 46 operators (all plus h.c.):

$2 D\left(d_{c}^{\dagger} d_{c} H^{\dagger} H B^{L}\right), 2 D\left(e_{c}^{\dagger} e_{c} H^{\dagger} H B^{L}\right), 2 D\left(d_{c}^{\dagger} d_{c} H^{\dagger} H G^{L}\right), 4 D\left(L^{\dagger} L H^{\dagger} H B^{L}\right)$, $4 D\left(Q^{\dagger} Q H^{\dagger} H B^{L}\right), 4 D\left(Q^{\dagger} Q H^{\dagger} H G^{L}\right), \quad D\left(d_{c}^{\dagger} u_{c}(H)^{2} B^{L}\right), \quad D\left(d_{c}^{\dagger} u_{c}(H)^{2} B^{R}\right)$, $D\left(d_{c}^{\dagger} u_{c}(H)^{2} G^{L}\right), \quad D\left(d_{c}^{\dagger} u_{c}(H)^{2} G^{R}\right), 2 D\left(u_{c}^{\dagger} u_{c} H^{\dagger} H B^{L}\right), 2 D\left(u_{c}^{\dagger} u_{c} H^{\dagger} H G^{L}\right)$, $2 D\left(d_{c}^{\dagger} d_{c} H^{\dagger} H W^{L}\right), 2 D\left(e_{c}^{\dagger} e_{c} H^{\dagger} H W^{L}\right), 6 D\left(L^{\dagger} L H^{\dagger} H W^{L}\right), 6 D\left(Q^{\dagger} Q H^{\dagger} H W^{L}\right)$,

$$
2 D\left(u_{c}^{\dagger} u_{c} H^{\dagger} H W^{L}\right), \quad D\left(d_{c}^{\dagger} u_{c}(H)^{2} W^{L}\right), \quad D\left(d_{c}^{\dagger} u_{c}(H)^{2} W^{R}\right) .
$$

Summing up all of the contributions gives a total of 181 dimension- 8 operators at $\mathcal{O}(D)$. If Hermitian conjugates are included, the total is 328 operators.

\subsection{Operators with derivatives: $\mathcal{O}\left(D^{2}\right)$}

We continue the procedure at $\mathcal{O}\left(D^{2}\right)$. There are now seven classes: $\mathcal{O}\left(D^{2}\left(H H^{\dagger}\right)^{3}\right)$, $\mathcal{O}\left(D^{2} X^{3}\right), \mathcal{O}\left(D^{2} \psi^{4}\right), \mathcal{O}\left(D^{2} \psi^{2} H^{3}\right), \mathcal{O}\left(D^{2} H^{2} X^{2}\right), \mathcal{O}\left(D^{2} \phi^{4} X\right)$ and $\mathcal{O}\left(D^{2} \psi^{2} H X\right):$

- $\mathcal{O}\left(D^{2} H^{3} H^{\dagger 3}\right)$. This class is the same as the complex scalar field we considered earlier. At $O\left(D^{2}\right)$ all partitions have only one Lorentz contraction.

- $\mathcal{O}\left(D^{2} X^{3}\right)$. Either all three field strengths are $X^{L}$ or $X^{R}$, or two are $X^{L}\left(X^{R}\right)$ with the third $X^{R}\left(X^{L}\right)$. In the first case, the partition $(1,1,0)$ is the only way to form a Lorentz invariant, and there is only one contraction. Of course, Bose symmetry or internal quantum numbers may forbid such a possibility, but even when allowed there is only one Lorentz contraction. In the mixed case, i.e $\left(X^{L}\right)^{2} X^{R}$, we can only form a Lorentz invariant if each of the $X^{L}$ are acted on with a derivative. This partition has only one contraction.

- $\mathcal{O}\left(D^{2} H^{2} X^{2}\right)$. More correctly, this class is $\mathcal{O}\left(D^{2} H H^{\dagger} X^{2}\right)$. In the case of a product of $X^{L} X^{R}$ gauge fields, the derivatives must act on the Higgses. The possibilities are $(2 ; 0 ; 0 ; 0),(0 ; 2 ; 0 ; 0)$, or $(1 ; 1 ; 0 ; 0)$, all of which admit a single Lorentz contraction 
regardless of how we stitch the $\mathrm{SU}(2)_{w}$ indices. For $\left(X^{L}\right)^{2}$ or $\left(X^{R}\right)^{2}$, two derivatives on a single Higgs no longer works. The Lorentz invariant possibilities are $(1 ; 1 ; 0,0)$, $(1 ; 0 ; 1,0)$ and $(0 ; 1 ; 1,0)$, all of which have a single contraction. However, if the two $X^{L, R}$ field strengths are different, i.e. $B^{L} W^{L}$, the number of partitions grows to 5 and $(1 ; 1 ; 0 ; 0)$ (now $D H^{\dagger} D H X_{i}^{L} X_{j}^{L}$ where $i$ and $j$ label different gauge groups) permits two contractions and therefore must be checked manually.

- $\mathcal{O}\left(D^{2} H^{4} X\right)=\mathcal{O}\left(D^{2} H^{2} H^{\dagger 2} X\right)$. The derivatives cannot act on $X$, and two derivatives on a single $H$ or $H^{\dagger}$ vanishes by the asymmetry of $X$, so the only possibilities are $(1,1 ; 0,0 ; 0),(0,0 ; 1,1 ; 0)$ and $(1,0 ; 1,0 ; 0)$, all of which can only be Lorentz contracted one way.

- $\mathcal{O}\left(D^{2} \psi^{4}\right)$. The four fermions must either have LL RR chirality or LLLL/RRRR. In the mixed chirality case there are three partitions. For $(1 ; 0 ; 1 ; 0)$ there is only one Lorentz contraction, so no issue. For $(1 ; 1 ; 0 ; 0)$ (in writing the partition this way we are allowing for the same chirality fermions to be different), $D \psi_{L, i} D \psi_{L, j}=$ $(0 \oplus 1,0 \oplus 1 \oplus 2)$ under $\left(\mathrm{SU}(2)_{R}, \mathrm{SU}(2)_{L}, \mathrm{SU}(2)_{w}\right)$ and $\psi_{R k} \psi_{R l}=(0 \oplus 1,0)$, so there are two different contractions. This means eq. (2.1) is not guaranteed and we have to look at the internal quantum numbers. For instance, if the two RH fermions (in this example) are identical, the $(1,0)$ (of $\left.\mathrm{SU}(2)_{R} \otimes \mathrm{SU}(2)_{L}\right)$ component of their product is forbidden unless they carry some other asymmetric quantum number. The partition $(0,0 ; 1,1)$ has the same issue. For LLLL/RRRR chirality operators, all partitions allow multiple contractions.

- $\mathcal{O}\left(D^{2} \psi^{2} H^{3}\right)$. This class is actually $\mathcal{O}\left(D^{2} \psi_{L i} \psi_{L i} H^{2} H^{\dagger}\right)$ (+ h.c.), with one of the two $\psi$ transforming as an $\mathrm{SU}(2)_{w}$ doublet (the two $\psi$ are then obviously different fields). There are many possible partitions, but the problematic one is $\psi_{L i} \psi_{L j} D H H D H^{\dagger}=$ $(0 ; 0 ; 1,0 ; 1)$. For this partition, the product of $\psi_{L i} \psi_{L j} H$ sits in the $(0,0 \oplus 1 ; 0 \oplus 1)$ representation of $\mathrm{SU}(2)_{R} \otimes \mathrm{SU}(2)_{R} \otimes \mathrm{SU}(2)_{w},{ }^{16}$ while the rest of the operator $D H D H^{\dagger}$ is in the $(0 \oplus 1,0 \oplus 1 ; 0 \oplus 1)$ representation. Combining the two pieces, we see that there are two Lorentz contractions for each $\mathrm{SU}(2)_{w}$ assignment (i.e. the $\mathrm{SU}(2)_{w}$ singlet part of $\psi_{L i} \psi_{L j} H$ can be either a Lorentz singlet or triplet when combining with $\left.D H D H^{\dagger}\right)$. Thus, we cannot guarantee eq. (2.1) will work for this class of operators.

- $\mathcal{O}\left(D^{2} \psi^{2} H X\right)$. As with the term above, both fermions must have the same chirality, represent different fields, and one must be an $\mathrm{SU}(2)_{w}$ doublet. Also like the term above, this term fails the criteria for eq. (2.1). The problematic partition is $D \psi_{L i} \psi_{L j} D H X$; both the derivative and non-derivative portions lie in Lorentz representation $\left(\frac{1}{2}, \frac{1}{2} \oplus \frac{3}{2}\right.$ ) (assuming $X^{L}$, the same logic goes through for $X^{R}$ ), and we can make Lorentz invariants by contracting either $\mathrm{SU}(2)_{L}$ possibility regardless of any $\mathrm{SU}(2)_{w}$ quantum numbers.

Recapping, four of the operator classes, $\mathcal{O}\left(D^{2} H^{3} H^{\dagger 3}\right), \mathcal{O}\left(D^{2} X^{3}\right), \mathcal{O}\left(D^{2} H^{2} X^{2}\right)$, and $\mathcal{O}\left(D^{2} H^{4} X\right)$ satisfy the criteria for eq. (2.1) and we can therefore automatically deter-

\footnotetext{
${ }^{16}$ Expanded out, this stands for $(0,0 ; 0) \oplus(0,0 ; 1) \oplus(0,1 ; 0) \oplus(0,1 ; 1)$.
} 
mine the number of invariants including IBP redundancies. ${ }^{17}$ Of the remaining classes, $\mathcal{O}\left(D^{2} \psi^{2} H^{3}\right)$ and $\mathcal{O}\left(D^{2} \psi^{2} H X\right)$ fail for any choice of fermion or gauge fields, while operators $\mathcal{O}\left(D^{2} \psi^{4}\right)$ may be okay depending on the internal symmetry quantum numbers of the fermions. For the operators classes where eq. (2.1) does not apply, the number of invariants can still be constructed, but it must be done manually by constructing the matrix of constraints from $\mathfrak{H}_{D, \text { SMEFT }}$ and determining its rank. For the classes where eq. (2.1) applies, we have the following two-derivative dimension- 8 operators.

Class $\mathcal{O}\left(\boldsymbol{D}^{2} \boldsymbol{H}^{\mathbf{3}} \boldsymbol{H}^{\dagger \mathbf{3}}\right)$. This class contains 2 self-Hermitian operators at $\mathcal{O}\left(D^{2} H^{6}\right)$

$$
2 D^{2}\left(H^{\dagger} H\right)^{3} .
$$

Class $\mathcal{O}\left(D^{2} X^{3}\right)$. After IBP redundancies have been considered, we find no operators of this type.

Class $\mathcal{O}\left(D^{2} H^{2} X^{2}\right)$. This class contains 4 self-Hermitian operators:

$$
D^{2}\left(H^{\dagger} H B^{L} B^{R}\right), \quad D^{2}\left(H^{\dagger} H G^{L} G^{R}\right), 2 D^{2}\left(H^{\dagger} H W^{L} W^{R}\right),
$$

and 5 operators with Hermitian conjugates

$$
\begin{gathered}
D^{2}\left(H^{\dagger} H\left(B^{L}\right)^{2}\right), \quad D^{2}\left(H^{\dagger} H\left(G^{L}\right)^{2}\right), \quad D^{2}\left(H^{\dagger} H\left(W^{L}\right)^{2}\right), \\
D^{2}\left(H^{\dagger} H B^{L} W^{L}\right), \quad D^{2}\left(H^{\dagger} H B^{R} W^{L}\right) .
\end{gathered}
$$

Class $\mathcal{O}\left(\boldsymbol{D}^{2} \boldsymbol{H}^{2} \boldsymbol{H}^{\dagger 2} \boldsymbol{X}\right)$. There is just one operator in this class (plus h.c.)

$$
D^{2}\left(\left(H^{\dagger} H\right)^{2} W^{L}\right)
$$

Next we can list the operators from the remaining classes. As stated above, the number of independent invariant operators in these classes must be determined manually. ${ }^{18}$

Class $\mathcal{O}\left(D^{2} \boldsymbol{\psi}^{4}\right)$. This class contains 39 self-Hermitian operators

$$
\begin{gathered}
2 D^{2}\left(d_{c}^{\dagger} d_{c}\right)^{2}, 2 D^{2}\left(u_{c}^{\dagger} u_{c}\right)^{2}, 4 D^{2}\left(Q^{\dagger} Q\right)^{2}, 2 D^{2}\left(L^{\dagger} L\right)^{2}, D^{2}\left(e_{c}^{\dagger} e_{c}\right)^{2}, \\
2 D^{2}\left(d_{c}^{\dagger} d_{c} e_{c}^{\dagger} e_{c}\right), 2 D^{2}\left(u_{c}^{\dagger} u_{c} e_{c}^{\dagger} e_{c}\right), 2 D^{2}\left(L^{\dagger} L e_{c}^{\dagger} e_{c}\right), 2 D^{2}\left(Q^{\dagger} Q e_{c}^{\dagger} e_{c}\right), \\
2 D^{2}\left(L^{\dagger} L d_{c}^{\dagger} d_{c}\right), 2 D^{2}\left(L^{\dagger} L u_{c}^{\dagger} u_{c}\right), 4 D^{2}\left(Q^{\dagger} Q d_{c}^{\dagger} d_{c}\right), 4 D^{2}\left(Q^{\dagger} Q u_{c}^{\dagger} u_{c}\right), \\
4 D^{2}\left(u_{c}^{\dagger} u_{c} d_{c}^{\dagger} d_{c}\right), \quad 4 D^{2}\left(Q^{\dagger} Q L^{\dagger} L\right)
\end{gathered}
$$

and 6 operators with Hermetian conjugate. These can be grouped into 4 which preserve baryon number

$$
2 D^{2}\left(L^{\dagger} e_{c}^{\dagger} Q d_{c}\right), 2 D^{2}\left(e_{c} L u_{c} Q\right)
$$

and 2 that do not.

$$
2 D^{2}\left(L^{\dagger} Q^{\dagger} u_{c} d_{c}\right)
$$

Operators in this class containing fermions of mixed chirality (LLRR) and multiple powers of the same field (i.e. $d_{c}^{2}$ ) actually satisfy the criteria for eq. (2.1) so the number of invariants can be determined automatically. This occurs because Fermi statistics limits the representations, thereby removing the troublesome extra Lorentz contractions.

\footnotetext{
${ }^{17}$ With the exception of $D^{2}\left(H^{\dagger} H B^{L, R} W^{L, R}\right)$.

${ }^{18}$ Although listed earlier, $D^{2}\left(H^{\dagger} H B^{L, R} W^{L, R}\right)$ is also in this operator set.
} 
Class $\mathcal{O}\left(D^{2} \psi^{2} H^{3}\right)$. We find 15 operators in this class (all plus h.c.):

$$
5 D^{2}\left(L e_{c} H\left(H^{\dagger}\right)^{2}\right), 5 D^{2}\left(Q d_{c} H\left(H^{\dagger}\right)^{2}\right), 5 D^{2}\left(Q u_{c} H^{2} H^{\dagger}\right) .
$$

Class $\mathcal{O}\left(D^{2} \psi^{2} \boldsymbol{H} \boldsymbol{X}\right)$. If the fermion's chirality is different than the gauge field, i.e $\psi_{L} \psi_{L} X^{R}$, there is only one contraction per partition and eq. (2.1) holds. For the 'same chirality' combinations, the invariants must be determined manually. We find 8 terms in this category, all + h.c.

$$
\begin{array}{cccc}
D^{2}\left(Q d_{c} H^{\dagger} F^{L}\right), & D^{2}\left(Q d_{c} H^{\dagger} G^{L}\right), & D^{2}\left(Q d_{c} H^{\dagger} W^{L}\right), & D^{2}\left(Q u_{c} H F^{L}\right), \\
D^{2}\left(Q u_{c} H G^{L}\right), & D^{2}\left(Q u_{c} H W^{L}\right), & D^{2}\left(L e_{c} H^{\dagger} F^{L}\right), & D^{2}\left(L e_{c} H^{\dagger} W^{L}\right),
\end{array}
$$

\subsection{Operators with derivatives: $\mathcal{O}\left(D^{3}\right)$}

As we increase the number of derivatives, the number of operator classes shrinks. At $\mathcal{O}\left(D^{3}\right)$ there are only two possibilities, $\mathcal{O}\left(D^{3} \psi^{3} X\right)$ and $\mathcal{O}\left(\psi^{3} H^{2}\right)$.

- $\mathcal{O}\left(D^{3} \psi^{2} X\right)$. To be Lorentz invariant the fermions must have opposite chirality. There are three ways to partition the derivatives, depending on whether the gauge field is $X^{L}$ or $X^{R}$. For $X^{L}$, one can have two derivatives on $\psi_{L}$ and one on $\psi_{R}$, two derivatives on $\psi_{L}$ and one on $X^{L}$, or one derivative on each. For each partition there is a single Lorentz contraction. As always, fermions with internal quantum numbers may have more invariants per contraction.

- $\mathcal{O}\left(D^{3} \psi^{2} H^{2}\right)$. More correctly, $\mathcal{O}\left(D^{3} \psi^{2} H H^{\dagger}\right)$, with the fermions having opposite chirality. There are 10 different partitions, most of which only allow one contraction, however the partitions $D \psi_{L} \psi_{R} D H D H^{\dagger}$ and $\psi_{L} D \psi_{R} D H D H^{\dagger}$ admit multiple. This class of operator therefore fails the criteria for eq. (2.1) and the number invariants must be calculated by hand.

Following the pattern of the $\mathcal{O}\left(D^{2}\right)$ operators, we list the operators in the classes satisfying eq. (2.1) first, then show the operators in classes that must be calculated manually.

Class $\mathcal{O}\left(D^{3} \psi^{2} X\right)$. After accounting for all redundancies, we find no operators of this type.

Class $\mathcal{O}\left(D^{3} \psi^{2} H^{2}\right)$. We find 14 self-hermitian operators of this type:

$$
\begin{gathered}
2 D^{3}\left(d_{c} d_{c}^{\dagger} H H^{\dagger}\right), 2 D^{3}\left(u_{c} u_{c}^{\dagger} H H^{\dagger}\right), 2 D^{3}\left(e_{c} e_{c}^{\dagger} H H^{\dagger}\right), \\
4 D^{3}\left(L L^{\dagger} H H^{\dagger}\right), 4 D^{3}\left(Q Q^{\dagger} H H^{\dagger}\right)
\end{gathered}
$$

\subsection{Operators with derivatives: $\mathcal{O}\left(D^{4}\right)$}

In this last category there are four classes: $\mathcal{O}\left(D^{4} X^{2}\right), \mathcal{O}\left(D^{4} H^{\dagger} H X\right), \mathcal{O}\left(D^{4} \psi^{2} H\right)$ and $\mathcal{O}\left(D^{4} H^{2} H^{\dagger 2}\right)$ :

- $\mathcal{O}\left(D^{4} X^{2}\right)$. Operators in this class are quadratic in fields, so there is only one way to partition the derivatives and only one contraction. 
- $\mathcal{O}\left(D^{4} H^{\dagger} H X\right)$. To be invariant, the field strength must be hypercharge. In this case, there are three possible partitions: $(2 ; 2 ; 0),(1 ; 2 ; 1)$, and $(2 ; 1 ; 1)$, each of which admits only one contraction.

- $\mathcal{O}\left(D^{4} \psi^{2} H\right)$. To maintain $\mathrm{SU}(2)_{w} \otimes \mathrm{U}(1)_{Y}$ invariance, these operators take the form of the four derivatives acting on the familiar $d=4$ Yukawa terms (i.e. $D^{4}\left(L e_{c} H\right)$ ). There are six partitions: $(2 ; 2 ; 0),(1 ; 1 ; 2),(2 ; 1 ; 1),(1 ; 2 ; 1),(2 ; 0 ; 2)$ and $(0 ; 2 ; 2)$, but only one contraction per partition.

- $\mathcal{O}\left(D^{4} H^{2} H^{\dagger 2}\right)$. These operators are analogous to the $\mathcal{O}\left(D^{4}\right)$ complex scalar operators we studied in section 2.1. As such, there are multiple contractions per derivative partition.

For the first three classes, we can rely on eq. (2.1) and find no operators after IBP redundancies are taken into account. For the last class, $\mathcal{O}\left(D^{4} H^{2} H^{\dagger 2}\right)$, the number of invariants must be derived manually. We find:

Class $\mathcal{O}\left(D^{4} H^{2} H^{\dagger 2}\right)$.

$$
3 D^{4}\left(H^{2} H^{\dagger 2}\right)
$$

\subsection{Total number of dimension- 8 operators}

The complete set of operators of dimension 8 is thus the sum of the sets above. In total, the number of operators we predict at dimension 8 by using eq. (2.1) for $N_{f}=1$ is $257+181+80+14+3=535$ operators. If Hermitian conjugates are included in the counting, the total comes to $471+328+115+14+3=931$ operators. Of the 535 operators, 46 violate baryon number. The fact that we need to determine the number of operators in some classes by hand makes it difficult to repeat this calculation for $N_{f}>1$.

\section{Discussion}

In this paper we proposed a method to incorporate derivatives into the Hilbert series, taking into account EOM and IBP redundancies. The method consists of two main steps. First, each spurion in the plethystic exponential is first dressed with derivatives, $\phi \rightarrow$ $\phi+D \phi+D^{2} \phi \cdots$ retaining only the symmetric pieces of $D^{m}$ when $m \geq 2$. The dressed PE is then used to form two separate series upon integration over the Haar measure: i.) the series of total (Lorentz and gauge) invariants, and ii.) the series of PE terms in the $\left(\frac{1}{2}, \frac{1}{2}\right)$ representation of the Lorentz group. The difference of these two series is the derivativeimproved Hilbert series. This treatment of derivatives and their redundancies was inspired in part by recent work on Hilbert series in $(0+1)$ spacetime dimension [2]. We show that this method of accounting for EOM and IBP overcounting is not foolproof - it fails whenever there are multiple possible Lorentz contractions for a given combination of derivatives, fields, and internal quantum number assignments. However, even with this limitation, the derivative-improved Hilbert series does successfully reproduce the previous counts of the dimension-6 and -7 SMEFT operators, and we thus conjecture that it is applicable for determining the majority of the dimension- 8 operators. We therefore calculate the 
$d=8, N_{f}=1$ list of operators by using a brute-force approach for the few cases where the derivative-improved series does not work. We also provide the list of $d=7$, arbitrary $N_{f}$ SMEFT operators in appendix D.

Since the Hilbert series technique and the associated mathematics have only recently been applied to the problem of calculating operators for effective field theories, there are a number of potential directions for future research. First, while our main focus in this work has been the SMEFT, the 'derivative-improved' Hilbert series technique we displayed will work for any EFT where the relevant degrees of freedom transform linearly under all symmetries of the theory. Similarly, though we have worked exclusively in four spacetime dimensions, the technique should apply to EFT in other spacetime dimensions as well, after appropriately changing the (Euclideanized) Lorentz symmetry. Another possible application is to include non-dynamical spurions (coupling matrices or vacuum expectation values) in addition to dynamical spurions (fields and their derivatives) in the Hilbert series. For example, we could enlarge the symmetry of the SMEFT to include quark and lepton flavor; the quark and leptons in the PEF would have to be modified to take into account their flavor transformations, but we could also add flavor bi-fundamental Yukawa matrices to the list of spurions in the PE. To account for the fact that Yukawa matrices are couplings and not fields, the Yukawa spurions would not be dressed with derivatives.

Finally, a complete implementation of derivatives accounting for IBP into the Hilbert series technique is a major goal of this line of research. This would remove the constraint of requiring a single Lorentz contraction per derivative partion (and quantum number assignment), and thus would allow the fully automated calculation of EFT operators to higher orders in derivatives. Such an implementation would also likely lead to a deeper understanding of why eq. (2.1) works so well for low-derivative operators.

Note added. A few weeks after this paper was put on the arxiv, the paper [56] appeared, which shows that the method for dealing with derivatives presented in this paper is not in fact completely correct. The method delineated in [56] seems to be a complete implementation of the derivative counting, and it finds 62 dimension- 8 operators (counting hermitian conjugates) that were missed by our method. The differences only occur in the 2- and 3-derivative operators; our results agree for the 0-, 1-, and 4-derivative operators. The method of [56] also gives a different counting of the 2-derivative dimension-7 operators for $N_{f}>1$.

\section{Acknowledgments}

We would like to thank the authors of ref. [2], Brian Henning, Xiaochuan Lu, Tom Melia and Hitoshi Murayama for kindly sharing their results with us over the last few months. We would also like to acknowledge Sekhar Chivukula, Brian Henning, Graham Kribs, Tom Melia and Veronica Sanz for useful discussions. The work of AM was partially supported by the National Science Foundation under Grant No. PHY-1417118. This research was supported in part by the Notre Dame Center for Research Computing through computing resources. 


\begin{tabular}{|c|cc|ccc|}
\hline & $\mathrm{SU}(2)_{R}$ & $\mathrm{SU}(2)_{L}$ & $\mathrm{SU}(3)_{c}$ & $\mathrm{SU}(2)_{w}$ & $\mathrm{U}(1)_{Y}$ \\
\hline$H$ & 0 & 0 & 0 & 2 & $\frac{1}{2}$ \\
$Q$ & 0 & $\frac{1}{2}$ & 3 & 2 & $\frac{1}{6}$ \\
$u_{c}$ & 0 & $\frac{1}{2}$ & $\overline{3}$ & 0 & $-\frac{2}{3}$ \\
$d_{c}$ & 0 & $\frac{1}{2}$ & $\overline{3}$ & 0 & $\frac{1}{3}$ \\
$L$ & 0 & $\frac{1}{2}$ & 0 & 2 & $-\frac{1}{2}$ \\
$e_{c}$ & 0 & $\frac{1}{2}$ & 0 & 0 & +1 \\
$B^{L}$ & 0 & 1 & 0 & 0 & 0 \\
$W^{L}$ & 0 & 1 & 0 & 3 & 0 \\
$G^{L}$ & 0 & 1 & 8 & 0 & 0 \\
\hline
\end{tabular}

Table 1. Fields and their quantum numbers of the SMEFT. The quantum numbers for conjugate fields can be derived by swapping $\mathrm{SU}(2)_{R}$ and $\mathrm{SU}(2)_{L}$ representations, reversing the sign of the $\mathrm{U}(1)_{Y}$ charge, and replacing $\mathrm{SU}(3)_{c}$ and $\mathrm{SU}(2)_{w}$ representations with their conjugates.

\section{A SMEFT fields and quantum numbers}

In table 1 we list the set of SMEFT spurions and their representations under $\operatorname{SU}(2)_{R}$, $\mathrm{SU}(2)_{L}, \mathrm{SU}(3)_{c}, \mathrm{SU}(2)_{w}, \mathrm{U}(1)_{Y}$.

\section{B From Hilbert series to operators: adding indices}

Asking for the invariants formed from two left-handed lepton doublets $L$ (of the same flavor), two $L^{\dagger}$ and two Higgses at dimension 8, the output of the Hilbert series is:

$$
2\left(L^{\dagger} L\right)^{2}\left(H^{\dagger} H\right)
$$

where the coefficient 2 indicates that there are two possible invariants. Since they are anticommuting fermionic fields, the two $L$ fields must form a totally antisymmetric combination of $(0,1 / 2 ; 0,1 / 2,-1 / 2)$ fields, where the numbers in parenthesis indicate the representation/quantum numbers under $\left(\mathrm{SU}(2)_{R}, \mathrm{SU}(2)_{L} ; \mathrm{SU}(3)_{c}, \mathrm{SU}(2)_{w}, \mathrm{U}(1)_{Y}\right)$ respectively:

$$
(0,1 / 2 ; 0,1 / 2,-1 / 2)_{\text {anti }}^{2}=(0,0 \oplus 1 ; 0,0 \oplus 1,-1)_{\text {anti }}=(0,0 ; 0,1,-1) \oplus(0,1 ; 0,0,-1),
$$

where antisymmetry kills the other two possibilities. The $\left(L^{\dagger}\right)^{2}$ piece is identical, except for $\mathrm{SU}(2)_{L} \rightarrow \mathrm{SU}(2)_{R}$ and hypercharge +1 instead of -1 . Combining the lepton pieces with the Higgs piece, we get

$$
\begin{aligned}
L^{2} \otimes L^{\dagger 2} \otimes\left(H^{\dagger} H\right)= & {[(0,0 ; 0,1,-1) \oplus(0,1 ; 0,0,-1)] \otimes[(0,0 ; 0,1,+1) \oplus(1,0 ; 0,0,+1)] } \\
& \otimes(0,0 ; 0,0 \oplus 1,0) .
\end{aligned}
$$

The two ways to make total invariants are now straightforward. The Lorentz triplet pieces from $L^{2}$ and $\left(L^{\dagger}\right)^{2}$ are in different $\mathrm{SU}(2)$ groups, so they cannot be joined to form an Lorentz invariant. The Lorentz singlet combination of $L^{2}$ and $\left(L^{\dagger}\right)^{2}$ can be either a $\mathrm{SU}(2)_{w}$ singlet, 
triplet, or quintuplet, and either the singlet or triplet can form an $\mathrm{SU}(2)_{w}$ invariant with the Higgs pair. Writing out the index contractions explicitly, we have the two independent operators

$$
\begin{gathered}
\epsilon_{A B}\left(\epsilon^{\alpha \beta} L_{\alpha, i} \tau_{j}^{A i} L_{\beta}^{j}\right)\left(\epsilon^{\dot{\alpha} \dot{\beta}} L_{\dot{\alpha}, k}^{\dagger} \tau_{m}^{B k} L_{\dot{\beta}}^{\dagger m}\right)\left(\epsilon^{x y} H_{x}^{\dagger} H_{y}\right) \\
\epsilon_{A B C}\left(\epsilon^{\alpha \beta} L_{\alpha, i} \tau_{j}^{A i} L_{\beta}^{j}\right)\left(\epsilon^{\dot{\alpha} \dot{\beta}} \epsilon^{k m} L_{\dot{\alpha}, k}^{\dagger} \tau_{m}^{B k} L_{\dot{\beta}}^{\dagger m}\right)\left(H_{x}^{\dagger} \tau_{y}^{C x} H^{y}\right) .
\end{gathered}
$$

Here the undotted (dotted) Greek indices refer to $\mathrm{SU}(2)_{L}\left(\mathrm{SU}(2)_{R}\right)$ indices, which are contracted via the epsilon tensor. We work in a convention where fundamental SU(2) indices are naturally lowered, and where the $(1,2)$ entry of $\epsilon^{\alpha \beta}$ is +1 (for any $\alpha, \beta$, both dotted and un-dotted). The $\tau^{A}$ matrices, with one lower and one upper index, are the canonical Pauli matrices. The Pauli matrices could be omitted in favor of symmetrized $\mathrm{SU}(2)_{w}$ indices.

\section{Hilbert series for a single scalar field}

Here we give the expanded expression for the derivative-improved Hilbert series for a single scalar field, $\mathfrak{H}_{\phi}$. We will use the complex variable $x$ to parameterize $\mathrm{SU}(2)_{L}$ and $y$ to parameterize $\mathrm{SU}(2)_{R}$. The Haar measure for the two $\mathrm{SU}(2)$ integrations is

$$
\int d \mu_{\mathrm{SU}(2)_{L}} d \mu_{\mathrm{SU}(2)_{R}}=\frac{1}{(2 \pi i)^{2}} \oint_{|x|=1} d x \frac{\left(x^{2}-1\right)}{x} \oint_{|y|=1} d y \frac{\left(y^{2}-1\right)}{y} .
$$

Following the discussion in section 2, the spurion for a single derivative on $\phi$ sits in the $\left(\frac{1}{2}, \frac{1}{2}\right)$ representation, the symmetric combination of two derivatives acting on $\phi$ sits in the $(1,1)$ representation, and the pattern continues for higher derivatives. For SU(2) parameterized by $x$, the characters for the smallest few representations are:

$$
\begin{array}{c|c}
1 & \text { singlet } \\
\left(x+\frac{1}{x}\right) & \text { doublet } \\
\left(1+x^{2}+\frac{1}{x^{2}}\right) & \text { triplet } \\
\left(x+x^{3}+\frac{1}{x}+\frac{1}{x^{3}}\right) & \text { quadruplet }
\end{array}
$$

Notice the characters are palindromic in $x \rightarrow \frac{1}{x}$ and the dimension of the representation is obtained by setting $x \rightarrow 1$.

Taking the characters from the table above, we can form the PE for the spurion $\phi$ dressed with derivatives:

$$
\begin{gathered}
\mathrm{PE}_{\phi}=\mathrm{PE}\left[\phi \left(1+D\left(x+\frac{1}{x}\right)\left(y+\frac{1}{y}\right)+D^{2}\left(1+x^{2}+\frac{1}{x^{2}}\right)\left(1+y^{2}+\frac{1}{y^{2}}\right)+\right.\right. \\
\left.\left.D^{3}\left(x+x^{3}+\frac{1}{x}+\frac{1}{x^{3}}\right)\left(y+y^{3}+\frac{1}{y}+\frac{1}{y^{3}}\right)+\mathcal{O}\left(D^{4}\right)+\cdots\right)\right] .
\end{gathered}
$$

To form the PE, each term in the expanded term bracketed above, $\phi, \phi D y x, \phi D \frac{y}{x}$, etc. must be separately plugged into eq. (1.2). It is possible the sum the powers of $D$ into a 
compact expression, similar to those shown in ref. [2] for $0+1$ dimensions. For scalars, the derivative sum is:

$$
\frac{\left(1-D^{2}\right) x^{2} y^{2} \phi}{(D x-y)(D y-x)(D-x y)(D x y-1)},
$$

and similar expressions can be obtained for fermions or field strengths. Plugging this expression into eq. (C.2), we can obtain the PE for scalars to all orders in $D$ - including EOM redundancies. However, as the expressions are quite lengthy and still contain IBP redundancies, we do not repeat them here. ${ }^{19}$ Finally, we find there is an alternative representation of scalar operators in terms of graphs. Specifically, each $\phi$ is a vertex and each contracted pair of derivatives is an edge. As such, the the number of operators with $n$ fields and $m$ derivatives (minus EOM) is equal to the number of loopless multigraphs with $n$ vertices and $m / 2$ edges [57].

\section{Dimension-7 operators for arbitrary $N_{f}$}

This appendix lists our prediction for dimension- 7 operators produced by the Hilbert series technique of eq. (2.1) for arbitrary $N_{f}$. To our knowledge, this has not been shown previously. First, we list the derivative-free operators (all plus h.c.):

$$
\begin{gathered}
\frac{1}{2}\left(N_{f}^{4}-N_{f}^{3}\right) Q\left(d_{c}^{\dagger}\right)^{2} e_{c} H^{\dagger}, \frac{1}{3}\left(N_{f}^{4}-N_{f}^{2}\right) L d_{c}^{3} H^{\dagger}, \quad N_{f}^{4} L\left(Q^{\dagger}\right)^{2} d_{c} H, \\
N_{f}^{4} L d_{c}^{2} u_{c} H, \quad N_{f}^{4} L e_{c}^{\dagger} u_{c}^{\dagger} d_{c} H, \frac{1}{2}\left(N_{f}^{2}+N_{f}\right) L^{2}\left(H^{\dagger} H\right) H^{2}, \\
\frac{1}{2}\left(N_{f}^{2}-N_{f}\right) L^{2} H^{2} B^{L}, \quad N_{f}^{2} L^{2} H^{2} W^{L}, N_{f}^{4} L^{2} Q^{\dagger} u_{c}^{\dagger} H, \\
2 N_{f}^{4} L^{2} Q d_{c} H, \frac{1}{2}\left(2 N_{f}^{4}+N_{f}^{2}\right) L^{3} e_{c} H .
\end{gathered}
$$

Next, there are the one and two derivative operators (all plus h.c.):

$$
\begin{gathered}
\frac{1}{6}\left(N_{f}^{4}+3 N_{f}^{3}+2 N_{f}^{2}\right) D e_{c}^{\dagger} d_{c}^{3}, \quad N_{f}^{2} D L e_{c}^{\dagger} H^{3}, \frac{1}{2}\left(N_{f}^{4}+N_{f}^{3}\right) D L Q^{\dagger} d_{c}^{2}, \\
\frac{1}{2}\left(N_{f}^{4}+N_{f}^{3}\right) D L^{2} d_{c} u_{c}^{\dagger}, \quad \frac{1}{2}\left(N_{f}^{2}+3 N_{f}\right) D^{2} L^{2} H^{2} .
\end{gathered}
$$

Adding up all of the contributions, the total number of dimension-7 operators for arbitrary $N_{f}$ is (not counting Hermitian conjugates):

$$
\frac{1}{6}\left(52 N_{f}^{4}+6 N_{f}^{3}+23 N_{f}^{2}+9 N_{f}\right)
$$

which is 15 for $N_{f}=1$ and 768 for $N_{f}=3$.

Open Access. This article is distributed under the terms of the Creative Commons Attribution License (CC-BY 4.0), which permits any use, distribution and reproduction in any medium, provided the original author(s) and source are credited.

\footnotetext{
${ }^{19}$ In $1+1$ dimensions, where the Lorentz group is just $\mathrm{U}(1)$, the all-orders PE is more compact $\frac{1}{1-\phi} \prod_{n=1}^{\infty} \frac{1}{\left(1-\phi x^{-n}\right)\left(1-\phi x^{n}\right)}$, where $x$ is the complex number parameterizing $\mathrm{U}(1)$.
} 


\section{References}

[1] L. Lehman and A. Martin, Hilbert Series for Constructing Lagrangians: expanding the phenomenologist's toolbox, Phys. Rev. D 91 (2015) 105014 [arXiv:1503.07537] [INSPIRE].

[2] B. Henning, X. Lu, T. Melia and H. Murayama, Hilbert series and operator bases with derivatives in effective field theories, arXiv:1507.07240 [INSPIRE].

[3] P. Pouliot, Molien function for duality, JHEP 01 (1999) 021 [hep-th/9812015] [INSPIRE].

[4] S. Benvenuti, B. Feng, A. Hanany and Y.-H. He, Counting BPS Operators in Gauge Theories: Quivers, Syzygies and Plethystics, JHEP 11 (2007) 050 [hep-th/0608050] [INSPIRE].

[5] F.A. Dolan, Counting BPS operators in N=4 SYM, Nucl. Phys. B 790 (2008) 432 [arXiv:0704.1038] [INSPIRE].

[6] J. Gray, A. Hanany, Y.-H. He, V. Jejjala and N. Mekareeya, SQCD: A Geometric Apercu, JHEP 05 (2008) 099 [arXiv:0803.4257] [INSPIRE].

[7] A. Hanany, N. Mekareeya and G. Torri, The Hilbert Series of Adjoint SQCD, Nucl. Phys. B 825 (2010) 52 [arXiv:0812.2315] [INSPIRE].

[8] Y. Chen and N. Mekareeya, The Hilbert series of U/SU SQCD and Toeplitz Determinants, Nucl. Phys. B 850 (2011) 553 [arXiv:1104.2045] [INSPIRE].

[9] A. Butti, D. Forcella, A. Hanany, D. Vegh and A. Zaffaroni, Counting Chiral Operators in Quiver Gauge Theories, JHEP 11 (2007) 092 [arXiv:0705.2771] [INSPIRE].

[10] B. Feng, A. Hanany and Y.-H. He, Counting gauge invariants: The plethystic program, JHEP 03 (2007) 090 [hep-th/0701063] [INSPIRE].

[11] D. Forcella, A. Hanany and A. Zaffaroni, Baryonic Generating Functions, JHEP 12 (2007) 022 [hep-th/0701236] [INSPIRE].

[12] S. Benvenuti, A. Hanany and N. Mekareeya, The Hilbert Series of the One Instanton Moduli Space, JHEP 06 (2010) 100 [arXiv: 1005.3026] [INSPIRE].

[13] A. Hanany, N. Mekareeya and S.S. Razamat, Hilbert Series for Moduli Spaces of Two Instantons, JHEP 01 (2013) 070 [arXiv:1205.4741] [INSPIRE].

[14] D. Rodríguez-Gómez and G. Zafrir, On the $5 d$ instanton index as a Hilbert series, Nucl. Phys. B 878 (2014) 1 [arXiv:1305.5684] [INSPIRE].

[15] A. Dey, A. Hanany, N. Mekareeya, D. Rodríguez-Gómez and R.-K. Seong, Hilbert Series for Moduli Spaces of Instantons on $\mathbb{C}^{2} / \mathbb{Z}_{n}$, JHEP 01 (2014) 182 [arXiv: 1309.0812] [INSPIRE].

[16] A. Hanany and R.-K. Seong, Hilbert series and moduli spaces of $k \mathrm{U}(N)$ vortices, JHEP 02 (2015) 012 [arXiv:1403.4950] [INSPIRE].

[17] L. Begin, C. Cummins and P. Mathieu, Generating functions for tensor products, hep-th/9811113 [INSPIRE].

[18] A. Hanany and R. Kalveks, Highest Weight Generating Functions for Hilbert Series, JHEP 10 (2014) 152 [arXiv:1408.4690] [INSPIRE].

[19] E.E. Jenkins and A.V. Manohar, Algebraic Structure of Lepton and Quark Flavor Invariants and CP-violation, JHEP 10 (2009) 094 [arXiv:0907.4763] [INSPIRE].

[20] A. Hanany, E.E. Jenkins, A.V. Manohar and G. Torri, Hilbert Series for Flavor Invariants of the Standard Model, JHEP 03 (2011) 096 [arXiv: 1010.3161] [INSPIRE]. 
[21] A. Merle and R. Zwicky, Explicit and spontaneous breaking of SU(3) into its finite subgroups, JHEP 02 (2012) 128 [arXiv:1110.4891] [INSPIRE].

[22] C. Grojean, E.E. Jenkins, A.V. Manohar and M. Trott, Renormalization Group Scaling of Higgs Operators and $\Gamma(h \rightarrow \gamma \gamma)$, JHEP 04 (2013) 016 [arXiv:1301.2588] [INSPIRE].

[23] E.E. Jenkins, A.V. Manohar and M. Trott, Renormalization Group Evolution of the Standard Model Dimension Six Operators I: Formalism and lambda Dependence, JHEP 10 (2013) 087 [arXiv:1308.2627] [INSPIRE].

[24] E.E. Jenkins, A.V. Manohar and M. Trott, Renormalization Group Evolution of the Standard Model Dimension Six Operators II: Yukawa Dependence, JHEP 01 (2014) 035 [arXiv:1310.4838] [INSPIRE].

[25] R. Alonso, E.E. Jenkins, A.V. Manohar and M. Trott, Renormalization Group Evolution of the Standard Model Dimension Six Operators III: Gauge Coupling Dependence and Phenomenology, JHEP 04 (2014) 159 [arXiv: 1312. 2014] [INSPIRE].

[26] J. Elias-Miró, J.R. Espinosa, E. Masso and A. Pomarol, Renormalization of dimension-six operators relevant for the Higgs decays $h \rightarrow \gamma \gamma, \gamma Z$, JHEP 08 (2013) 033 [arXiv:1302.5661] [INSPIRE].

[27] J. Elias-Miro, J.R. Espinosa, E. Masso and A. Pomarol, Higgs windows to new physics through $D=6$ operators: constraints and one-loop anomalous dimensions, JHEP 11 (2013) 066 [arXiv:1308.1879] [INSPIRE].

[28] R. Alonso, H.-M. Chang, E.E. Jenkins, A.V. Manohar and B. Shotwell, Renormalization group evolution of dimension-six baryon number violating operators, Phys. Lett. B 734 (2014) 302 [arXiv: 1405.0486] [INSPIRE].

[29] R. Alonso, E.E. Jenkins and A.V. Manohar, Holomorphy without Supersymmetry in the Standard Model Effective Field Theory, Phys. Lett. B 739 (2014) 95 [arXiv:1409.0868] [INSPIRE].

[30] M. Trott, On the consistent use of Constructed Observables, JHEP 02 (2015) 046 [arXiv: 1409.7605] [INSPIRE].

[31] B. Henning, X. Lu and H. Murayama, How to use the Standard Model effective field theory, JHEP 01 (2016) 023 [arXiv: 1412.1837] [INSPIRE].

[32] S. Willenbrock and C. Zhang, Effective Field Theory Beyond the Standard Model, Ann. Rev. Nucl. Part. Sci. 64 (2014) 83 [arXiv:1401.0470] [INSPIRE].

[33] J. Elias-Miro, J.R. Espinosa and A. Pomarol, One-loop non-renormalization results in EFTs, Phys. Lett. B 747 (2015) 272 [arXiv:1412.7151] [INSPIRE].

[34] R.S. Gupta, A. Pomarol and F. Riva, BSM Primary Effects, Phys. Rev. D 91 (2015) 035001 [arXiv: 1405.0181] [INSPIRE].

[35] G.F. Giudice, C. Grojean, A. Pomarol and R. Rattazzi, The Strongly-Interacting Light Higgs, JHEP 06 (2007) 045 [hep-ph/0703164] [INSPIRE].

[36] M. Duehrssen-Debling et al., Higgs Basis, Proposal for an EFT basis choice for LHC HXSWG, LHC Higgs Cross section Working Group 2, LHCHXSWG-INT-2015-001.

[37] C. Cheung and C.-H. Shen, Nonrenormalization Theorems without Supersymmetry, Phys. Rev. Lett. 115 (2015) 071601 [arXiv:1505.01844] [INSPIRE]. 
[38] L. Berthier and M. Trott, Consistent constraints on the Standard Model Effective Field Theory, arXiv:1508.05060 [INSPIRE].

[39] C.-W. Chiang and R. Huo, Standard Model Effective Field Theory: Integrating out a Generic Scalar, JHEP 09 (2015) 152 [arXiv: 1505.06334] [INSPIRE].

[40] R. Huo, Standard Model Effective Field Theory: Integrating out Vector-Like Fermions, JHEP 09 (2015) 037 [arXiv: 1506.00840] [INSPIRE].

[41] R. Huo, Effective Field Theory of Integrating out Sfermions in the MSSM: Complete One-Loop Analysis, arXiv: 1509.05942 [INSPIRE].

[42] A. Drozd, J. Ellis, J. Quevillon and T. You, Comparing EFT and Exact One-Loop Analyses of Non-Degenerate Stops, JHEP 06 (2015) 028 [arXiv: 1504.02409] [INSPIRE].

[43] S. Weinberg, Baryon and Lepton Nonconserving Processes, Phys. Rev. Lett. 43 (1979) 1566 [INSPIRE].

[44] W. Buchmüller and D. Wyler, Effective Lagrangian Analysis of New Interactions and Flavor Conservation, Nucl. Phys. B 268 (1986) 621 [INSPIRE].

[45] B. Grzadkowski, M. Iskrzynski, M. Misiak and J. Rosiek, Dimension-Six Terms in the Standard Model Lagrangian, JHEP 10 (2010) 085 [arXiv:1008.4884] [INSPIRE].

[46] L.F. Abbott and M.B. Wise, The Effective Hamiltonian for Nucleon Decay, Phys. Rev. D 22 (1980) 2208 [inSPIRE].

[47] L. Lehman, Extending the Standard Model Effective Field Theory with the Complete Set of Dimension-7 Operators, Phys. Rev. D 90 (2014) 125023 [arXiv:1410.4193] [INSPIRE].

[48] G.J. Gounaris, J. Layssac and F.M. Renard, Addendum to off-shell structure of the anomalous $Z$ and gamma selfcouplings, Phys. Rev. D 65 (2002) 017302 [INSPIRE].

[49] C. Degrande, A basis of dimension-eight operators for anomalous neutral triple gauge boson interactions, JHEP 02 (2014) 101 [arXiv:1308.6323] [INSPIRE].

[50] H.D. Politzer, Power Corrections at Short Distances, Nucl. Phys. B 172 (1980) 349 [INSPIRE].

[51] H. Georgi, On-shell effective field theory, Nucl. Phys. B 361 (1991) 339 [INSPIRE].

[52] C. Arzt, Reduced effective Lagrangians, Phys. Lett. B 342 (1995) 189 [hep-ph/9304230] [INSPIRE].

[53] C. Grosse-Knetter, Effective Lagrangians with higher derivatives and equations of motion, Phys. Rev. D 49 (1994) 6709 [hep-ph/9306321] [INSPIRE].

[54] H. Simma, Equations of motion for effective Lagrangians and penguins in rare $B$ decays, Z. Phys. C 61 (1994) 67 [hep-ph/9307274] [INSPIRE].

[55] K. Hagiwara, S. Ishihara, R. Szalapski and D. Zeppenfeld, Low-energy effects of new interactions in the electroweak boson sector, Phys. Rev. D 48 (1993) 2182 [INSPIRE].

[56] B. Henning, X. Lu, T. Melia and H. Murayama, 2, 84, 30, 993, 560, 15456, 11962, 261485, ...: Higher dimension operators in the SM EFT, arXiv:1512.03433 [INSPIRE].

[57] J.L. Gross, J. Yellen and P. Zhang, Handbook of Graph Theory, second edition, Chapman \& Hall/CRC (2013). 